\title{
EXISTENCE OF LIMITING DISTRIBUTION FOR AFFINE PROCESSES
}

\author{
PENG JIN*, JONAS KREMER, AND BARBARA RÜDIGER
}

\begin{abstract}
In this paper, sufficient conditions are given for the existence of limiting distribution of a conservative affine process on the canonical state space $\mathbb{R}_{\geqslant 0}^{m} \times \mathbb{R}^{n}$, where $m, n \in \mathbb{Z}_{\geqslant 0}$ with $m+n>0$. Our main theorem extends and unifies some known results for OU-type processes on $\mathbb{R}^{n}$ and one-dimensional CBI processes (with state space $\mathbb{R} \geqslant 0$ ). To prove our result, we combine analytical and probabilistic techniques; in particular, the stability theory for ODEs plays an important role.
\end{abstract}

\section{Introduction}

Let $D:=\mathbb{R}_{\geqslant 0}^{m} \times \mathbb{R}^{n}$, where $m, n \in \mathbb{Z}_{\geqslant 0}$ with $m+n>0$. Roughly speaking, an affine process with state space $D$ is a time-homogeneous Markov process $\left(X_{t}\right)_{t \geqslant 0}$ taking values in $D$, whose log-characteristic function depends in an affine way on the initial value of the process, that is, there exist functions $\phi, \psi=\left(\psi_{1}, \ldots, \psi_{m+n}\right)$ such that

$$
\mathbb{E}\left[\mathrm{e}^{\left\langle u, X_{t}\right\rangle} \mid X_{0}=x\right]=\mathrm{e}^{\phi(t, u)+\langle\psi(t, u), x\rangle},
$$

for all $u \in \mathbb{i}^{m+n}, t \geqslant 0$ and $x \in D$. The general theory of affine processes was initiated by Duffie, Pan and Singleton [9] and further developed by Duffie, Filipović, and Schachermayer [8]. In the seminal work of Duffie et al. [8], several fundamental properties of affine processes on the canonical state space $D$ were established. In particular, the generator of $D$-valued affine processes is completely characterized through a set of admissible parameters, and the associated generalized Riccati equations for $\phi$ and $\psi$ are introduced and studied. The results of [8] were further complemented by many subsequent developments, see, e.g., 1, 3, 4, 7, 11, 14, 16, 18.

Affine processes have found a wide range of applications in finance, mainly due to their computational tractability and modeling flexibility. Many popular models in finance, such as the models of Cox et al. [5, Heston [13] and Vasicek 25, are of affine type. Moreover, from the theoretical point of view, the concept of affine processes enables a unified treatment of two very important classes of continuous-time

Date: December 14, 2018.

2010 Mathematics Subject Classification. Primary 60J25; Secondary 60G10.

Key words and phrases. affine process, limiting distribution, stationary distribution, generalized Riccati equation.

${ }^{*}$ Peng Jin is partially supported by the STU Scientific Research Foundation for Talents (No. NTF18023). 
Markov processes: OU-type processes on $\mathbb{R}^{n}$ and CBI (continuous-state branching processes with immigration) processes on $\mathbb{R}_{\geqslant 0}^{m}$.

In this paper, we are concerned with the following question: when does an affine process converge in law to a limit distribution? This problem has already been dealt with in the following situations:

- Sato and Yamazato 23] provided conditions under which an OU-type process on $\mathbb{R}^{n}$ converges in law to a limit distribution, and they identified this type of limit distributions with the class of operator self-decomposable distributions of Urbanik [24;

- without a proof, Pinsky [22] announced the existence of a limit distribution for one-dimensional CBI processes, under a mean-reverting condition and the existence of the log-moment of the Lévy measure from the immigration mechanism. A recent proof appeared in [20, Theorem 3.20 and Corollary 3.21] (see also [15, Theorem 3.16]). A stronger form of this result can be found in [17, Theorem 2.6];

- Glasserman and Kim [12] proved that affine diffusion processes on $\mathbb{R}_{\geqslant 0}^{m} \times \mathbb{R}^{n}$ introduced by Dai and Singleton [6] have limiting stationary distributions and characterized these limits;

- Barczy, Döring, Li, and Pap [2] showed stationarity of an affine two-factor model on $\mathbb{R}_{\geqslant 0} \times \mathbb{R}$, with one component being the $\alpha$-root process.

Our motivation for this paper is twofold. On the one hand, we would like to formulate a general result for affine processes with state space $D=\mathbb{R}_{\geqslant 0}^{m} \times \mathbb{R}^{n}$, which unifies the above mentioned results; on the other hand, our result should also provide new results for the unsolved cases where $D=\mathbb{R}_{\geqslant 0}^{m}(m \geqslant 2)$ and $D=\mathbb{R}_{\geqslant 0}^{m} \times \mathbb{R}^{n}$ $(m \geqslant 1, n \geqslant 1)$. As our main result (see Theorem 2.6 below), we give sufficient conditions such that an affine process $X$ with state space $D=\mathbb{R}_{\geqslant 0}^{m} \times \mathbb{R}^{n}$ converges in law to a limit distribution as time goes to infinity, and we also identify this limit through its characteristic function. Using a similar argument as in [15, we will show that the limit distribution is the unique stationary distribution for $X$.

The rest of this paper is organized as follows. In Section 2 we recall some definitions regarding affine processes and present our main theorem, whose proof we defer to Section 4. In Section 3 we deal with the large time behavior of the function $\psi$ and show that $\psi(t, u)$ converges exponentially fast to 0 as $t$ goes to infinity. Finally, we prove our main theorem in Section 4.

\section{Preliminaries and main result}

2.1. Notation. Let $\mathbb{N}, \mathbb{Z}_{\geqslant 0}, \mathbb{R}$ denote the sets of positive integers, non-negative integers and real numbers, respectively. Let $\mathbb{R}^{d}$ be the $d$-dimensional $(d \geqslant 1)$ Euclidean space and define

$$
\mathbb{R}_{\geqslant 0}^{d}:=\left\{x \in \mathbb{R}^{d}: x_{i} \geqslant 0, i=1, \ldots, d\right\}
$$

and

$$
\mathbb{R}_{>0}^{d}:=\left\{x \in \mathbb{R}^{d}: x_{i}>0, i=1, \ldots, d\right\}
$$


For $x, y \in \mathbb{R}$, we write $x \wedge y:=\min \{x, y\}$. By $\langle\cdot, \cdot\rangle$ and $\|x\|$ we denote the inner product on $\mathbb{R}^{d}$ and the induced Euclidean norm of a vector $x \in \mathbb{R}^{d}$, respectively. For a $d \times d$-matrix $A=\left(a_{i j}\right)$, we write $A^{\top}$ for the transpose of $A$ and define $\|A\|:=\left(\operatorname{trace}\left(A^{\top} A\right)\right)^{1 / 2}$. Let $\mathbb{C}^{d}$ be the space that consists of $d$-tuples of complex numbers. We define the following subsets of $\mathbb{C}^{d}$ :

$$
\mathbb{C}_{\leqslant 0}^{d}:=\left\{u \in \mathbb{C}^{d}: \operatorname{Re} u_{i} \leqslant 0, i=1, \ldots, d\right\}
$$

and

$$
\mathrm{i} \mathbb{R}^{d}:=\left\{u \in \mathbb{C}^{d}: \operatorname{Re} u_{i}=0, i=1, \ldots, d\right\} .
$$

The following sets of matrices are of particular importance in this work :

- $\mathbb{M}_{d}^{-}$which stands for the set of real $d \times d$ matrices all of whose eigenvalues have strictly negative real parts. Note that $A \in \mathbb{M}_{d}^{-}$if and only if $\|\exp \{t A\}\| \rightarrow 0$ as $t \rightarrow \infty$;

- $\mathbb{S}_{d}^{+}$(resp. $\mathbb{S}_{d}^{++}$) which stands for the set of all symmetric and positive semidefinite (resp. positive definite) real $d \times d$ matrices.

If $A=\left(a_{i j}\right)$ is a $d \times d$-matrix, $b=\left(b_{1}, \ldots, b_{d}\right) \in \mathbb{R}^{d}$ and $\mathcal{I}, \mathcal{J} \subset\{1, \ldots, d\}$, we write $A_{\mathcal{I} \mathcal{J}}:=\left(a_{i j}\right)_{i \in \mathcal{I}, j \in \mathcal{J}}$ and $b_{\mathcal{I}}:=\left(b_{i}\right)_{i \in \mathcal{I}}$.

Let $U$ be an open set or the closure of an open set in $\mathbb{R}^{d}$. We introduce the following function spaces: $C^{k}(U), C_{c}^{k}(U)$, and $C^{\infty}(U)$ which denote the sets of $\mathbb{C}$-valued functions on $U$ that are $k$-times continuously differentiable, that are $k$-times continuously differentiable with compact support, and that are smooth, respectively. The Borel $\sigma$-Algebra on $U$ will be denoted by $\mathcal{B}(U)$.

Throughout the rest of this paper, let $D:=\mathbb{R}_{\geqslant 0}^{m} \times \mathbb{R}^{n}$, where $m, n \in \mathbb{Z}_{\geqslant 0}$ with $m+n>0$. Note that $m$ or $n$ may be 0 . The set $D$ will act as the state space of affine processes we are about to consider. The total dimension of $D$ is denoted by $d=m+n$. We write $\mathcal{B}_{b}(D)$ for the Banach space of bounded real-valued Borel measurable functions $f$ on $D$ with norm $\|f\|_{\infty}:=\sup _{x \in D}|f(x)|$.

For $D$, we write

$$
I=\{1, \ldots, m\} \quad \text { and } \quad J=\{m+1, \ldots, m+n\}
$$

for the index sets of the $\mathbb{R}_{\geqslant 0}^{m}$-valued components and the $\mathbb{R}^{n}$-valued components, respectively. Define

$$
\mathcal{U}:=\mathbb{C}_{\leqslant 0}^{m} \times \mathrm{i} \mathbb{R}^{n}=\left\{u \in \mathbb{C}^{d}: \operatorname{Re} u_{I} \leqslant 0, \quad \operatorname{Re} u_{J}=0\right\} .
$$

Note that $\mathcal{U}$ is the set of all $u \in \mathbb{C}^{d}$, for which $x \mapsto \exp \{\langle u, x\rangle\}$ is a bounded function on $D$.

Further notation is introduced in the text.

2.2. Affine processes on the canonical state space. Affine processes on the canonical state space $D=\mathbb{R}_{\geqslant 0}^{m} \times \mathbb{R}^{n}$ have been systematically studied in the well-known work [8]. We remark that affine processes considered in $[\underline{8}$ are in full generality and are allowed to have explosions or killings. In contrast to [8, in this paper we restrict ourselves to conservative affine processes. In terms of terminology and notation, we mainly follow, instead of [8], the paper by Keller-Ressel and Mayerhofer [16], where only the conservative case was considered. 
Let us start with a time-homogeneous and conservative Markov process with state space $D$ and semigroup $\left(P_{t}\right)$ acting on $\mathcal{B}_{b}(D)$, that is,

$$
P_{t} f(x)=\int_{D} f(\xi) p_{t}(x, d \xi), \quad f \in \mathcal{B}_{b}(D) .
$$

Here $p_{t}(x, \cdot)$ denotes the transition kernel of the Markov process. We assume that $p_{0}(x,\{x\})=1$ and $p_{t}(x, D)=1$ for all $t \geqslant 0, x \in D$.

Let $\left(X,\left(\mathbb{P}_{x}\right)_{x \in D}\right)$ be the canonical realization of $\left(P_{t}\right)$ on $\left(\Omega, \mathcal{F},\left(\mathcal{F}_{t}\right)_{t \geqslant 0}\right)$, where $\Omega$ is the set of all càdlàg paths in $D$ and $X_{t}(\omega)=\omega(t)$ for $\omega \in \Omega$. Here $\left(\mathcal{F}_{t}\right)_{t \geqslant 0}$ is the filtration generated by $X$ and $\mathcal{F}=\bigvee_{t \geqslant 0} \mathcal{F}_{t}$. The probability measure $\mathbb{P}_{x}$ on $\Omega$ represents the law of the Markov process $\left(X_{t}\right)_{t \geqslant 0}$ started at $x$, i.e., it holds that $X_{0}=x, \mathbb{P}_{x}$-almost surely. The following definition is taken from [16, Definition $2.2]$.

Definition 2.1. The Markov process $X$ is called affine with state space $D$, if its transition kernel $p_{t}(x, A)=\mathbb{P}_{x}\left(X_{t} \in A\right)$ satisfies the following:

(i) it is stochastically continuous, that is, $\lim _{s \rightarrow t} p_{s}(x, \cdot)=p_{t}(x, \cdot)$ weakly for all $t \geqslant 0, x \in D$, and

(ii) there exist functions $\phi: \mathbb{R}_{\geqslant 0} \times \mathcal{U} \rightarrow \mathbb{C}$ and $\psi: \mathbb{R}_{\geqslant 0} \times \mathcal{U} \rightarrow \mathbb{C}^{d}$ such that

$$
\int_{D} \mathrm{e}^{\langle u, \xi\rangle} p_{t}(x, \mathrm{~d} \xi)=\mathbb{E}_{x}\left[\mathrm{e}^{\left\langle X_{t}, u\right\rangle}\right]=\exp \{\phi(t, u)+\langle x, \psi(t, u)\rangle\}
$$

for all $t \geqslant 0, x \in D$ and $u \in \mathcal{U}$, where $\mathbb{E}_{x}$ denotes the expectation with respect to $\mathbb{P}_{x}$.

The stochastic continuity in (i) and the affine property in (ii) together imply the following regularity of the functions $\phi$ and $\psi$ (see [18, Theorem 5.1]), i.e., the right-hand derivatives

$$
F(u):=\left.\frac{\partial}{\partial t} \phi(t, u)\right|_{t=0+} \text { and } R(u):=\left.\frac{\partial}{\partial t} \psi(t, u)\right|_{t=0+}
$$

exist for all $u \in \mathcal{U}$, and are continuous at $u=0$. Moreover, according to 8 , Proposition 7.4], the functions $\phi$ and $\psi$ satisfy the semi-flow property:

$$
\phi(t+s, u)=\phi(t, u)+\phi(s, \psi(t, u)) \quad \text { and } \quad \psi(t+s, u)=\psi(s, \psi(t, u)),
$$

for all $t, s \geqslant 0$ with $(t+s, u) \in \mathbb{R}_{\geqslant 0} \times \mathcal{U}$.

Definition 2.2. We call $(a, \alpha, b, \beta, m, \mu)$ a set of admissible parameters for the state space $D$ if

(i) $a \in \mathbb{S}_{d}^{+}$and $a_{k l}=0$ for all $k \in I$ or $l \in I$;

(ii) $\alpha=\left(\alpha_{1}, \ldots, \alpha_{m}\right)$ with $\alpha_{i}=\left(\alpha_{i, k l}\right)_{1 \leqslant k, l \leqslant d} \in \mathbb{S}_{d}^{+}$

$$
\text { and } \alpha_{i, k l}=0 \text { if } k \in I \backslash\{i\} \text { or } l \in I \backslash\{i\} \text {; }
$$

(iii) $m$ is a Borel measure on $D \backslash\{0\}$ satisfying

$$
\int_{D \backslash\{0\}}\left(1 \wedge\|\xi\|^{2}+\sum_{i \in I}\left(1 \wedge \xi_{i}\right)\right) m(\mathrm{~d} \xi)<\infty
$$


(iv) $\mu=\left(\mu_{1}, \ldots, \mu_{m}\right)$ where every $\mu_{i}$ is a Borel measure on $D \backslash\{0\}$ satisfying

$$
\int_{D \backslash\{0\}}\left(\|\xi\| \wedge\|\xi\|^{2}+\sum_{k \in I \backslash\{i\}} \xi_{k}\right) \mu_{i}(\mathrm{~d} \xi)<\infty .
$$

(v) $b \in D$;

(vi) $\beta=\left(\beta_{k i}\right) \in \mathbb{R}^{d \times d}$ with $\beta_{k i}-\int_{D \backslash\{0\}} \xi_{k} \mu_{i}(\mathrm{~d} \xi) \geqslant 0$ for all $i \in I$ and $k \in I \backslash\{i\}$, and $\beta_{k i}=0$ for all $k \in I$ and $i \in J$;

We remark that our definition of admissible parameters is a special case of [8, Definition 2.6], since we require here that the parameters corresponding to killing are constant 0 ; moreover, the condition in (iv) is also stronger as usual, i.e., we assume that the first moment of $\mu_{i}$ 's exists, which, by [8, Lemma 9.2], implies that the affine process under consideration is conservative. However, we should remind the reader that (2.4) is not a necessary condition for conservativeness. In fact, an example of a conservative affine process on $\mathbb{R}_{\geqslant 0}$, which violates (2.4), is provided in [21, Section 3].

We write $\psi=\left(\psi^{I}, \psi^{J}\right) \in \mathbb{C}^{m} \times \mathbb{C}^{n}$, where $\psi^{I}=\left(\psi_{1}, \ldots, \psi_{m}\right)^{\top}$ and $\psi^{J}=$ $\left(\psi_{m+1}, \ldots, \psi_{m+n}\right)^{\top}$. Recall that $R=\left(R_{1}, \ldots, R_{d}\right)^{\top}: \mathcal{U} \rightarrow \mathbb{C}^{d}$ is given in (2.2). Define $R^{I}:=\left(R_{1}, \ldots, R_{m}\right)^{\top}: \mathcal{U} \rightarrow \mathbb{C}^{m}$. For $u \in \mathcal{U}$, we will often write $u=(v, w) \in \mathbb{C}_{\leqslant 0}^{m} \times \mathrm{i} \mathbb{R}^{n}$.

The next result is due to [8, Theorem 2.7].

Theorem 2.3. Let $(a, \alpha, b, \beta, m, \mu)$ be a set of admissible parameters in the sense of Definition 2.2. Then there exists a (unique) conservative affine process $X$ with state space $D$ such that its infinitesimal generator $\mathcal{A}$ operating on a function $f \in$ $C_{c}^{2}(D)$ is given by

$$
\begin{aligned}
\mathcal{A} f(x)= & \sum_{k, l=1}^{d}\left(a_{k l}+\sum_{i=1}^{m} \alpha_{i, k l} x_{i}\right) \frac{\partial^{2} f(x)}{\partial x_{k} \partial x_{l}}+\langle b+\beta x, \nabla f(x)\rangle \\
& +\int_{D \backslash\{0\}}\left(f(x+\xi)-f(x)-\left\langle\nabla_{J} f(x), \xi_{J}\right\rangle \mathbb{1}_{\{\|\xi\| \leqslant 1\}}(\xi)\right) m(\mathrm{~d} \xi) \\
& +\sum_{i=1}^{m} x_{i} \int_{D \backslash\{0\}}(f(x+\xi)-f(x)-\langle\nabla f(x), \xi\rangle) \mu_{i}(\mathrm{~d} \xi)
\end{aligned}
$$

where $x \in D, \nabla_{J}:=\left(\partial_{x_{k}}\right)_{k \in J}$. Moreover, (2.1) holds for some functions $\phi(t, u)$ and $\psi(t, u)$ that are uniquely determined by the generalized Riccati differential equations: for each $u=(v, w) \in \mathbb{C}_{\leqslant 0}^{m} \times \mathbb{i}^{n}$,

$$
\begin{aligned}
\partial_{t} \phi(t, u) & =F(\psi(t, u)), \quad \phi(0, u)=0, \\
\partial_{t} \psi^{I}(t, u) & =R^{I}\left(\psi^{I}(t, u), \mathrm{e}^{\beta_{J}^{\top} t} w\right), \quad \psi^{I}(0, u)=v \\
\psi^{J}(t, u) & =\mathrm{e}^{\beta_{J J}^{\top}} w
\end{aligned}
$$


where

$$
F(u)=\langle u, a u\rangle+\langle b, u\rangle+\int_{D \backslash\{0\}}\left(\mathrm{e}^{\langle u, \xi\rangle}-1-\left\langle u_{J}, \xi_{J}\right\rangle \mathbb{1}_{\{\|\xi\| \leqslant 1\}}(\xi)\right) m(\mathrm{~d} \xi)
$$

and $R^{I}=\left(R_{1}, \ldots, R_{m}\right)$ with

$$
R_{i}(u)=\left\langle u, \alpha_{i} u\right\rangle+\sum_{k=1}^{d} \beta_{k i} u_{k}+\int_{D \backslash\{0\}}\left(\mathrm{e}^{\langle u, \xi\rangle}-1-\langle u, \xi\rangle\right) \mu_{i}(\mathrm{~d} \xi), \quad i \in I .
$$

Remark 2.4. If an affine process $X$ with state space $D$ and a set of admissible parameters $(a, \alpha, b, \beta, m, \mu)$ satisfy a relation as in Theorem 2.3 , then we say that $X$ is an affine process with admissible parameters $(a, \alpha, b, \beta, m, \mu)$.

The following lemma is a consequence of the condition (iv) in Definition 2.2 .

Lemma 2.5. Let $X$ be an affine process with state space $D$ and admissible parameters $(a, \alpha, b, \beta, m, \mu)$. Let $R$ and $\psi$ be as in Theorem 2.3. For each $i \in I$ it holds that $R_{i} \in C^{1}(\mathcal{U})$ and $\psi_{i} \in C^{1}\left(\mathbb{R}_{\geqslant 0} \times \mathcal{U}\right)$.

To see that Lemma 2.5 is true, we only need to apply Lemmas 5.3 and 6.5 of [8.

2.3. Main result. Our main result of this paper is the following.

Theorem 2.6. Let $X$ be an affine process with state space $\mathbb{R}_{\geqslant 0}^{m} \times \mathbb{R}^{n}$ and admissible parameters $(a, \alpha, b, \beta, m, \mu)$ in the sense of Definition 2.2. If

$$
\beta \in \mathbb{M}_{d}^{-} \quad \text { and } \quad \int_{\{\|\xi\|>1\}} \log \|\xi\| m(\mathrm{~d} \xi)<\infty,
$$

then the law of $X_{t}$ converges weakly to a limiting distribution $\pi$, which is independent of $X_{0}$ and whose characteristic function is given by

$$
\int_{D} \mathrm{e}^{\langle u, x\rangle} \pi(\mathrm{d} x)=\exp \left\{\int_{0}^{\infty} F(\psi(s, u)) \mathrm{d} s\right\}, \quad u \in \mathcal{U} .
$$

Moreover, the limiting distribution $\pi$ is the unique stationary distribution for $X$.

Remark 2.7. In virtue of the definition of admissible parameters, we can write $\beta \in \mathbb{R}^{d \times d}$ in the following way:

$$
\beta=\left(\begin{array}{c:c}
\beta_{I I} & 0 \\
\hdashline \beta_{J I} & \beta_{J J}
\end{array}\right),
$$

where $\beta_{I I} \in \mathbb{R}^{m \times m}, \beta_{J I} \in \mathbb{R}^{n \times m}$ and $\beta_{J J} \in \mathbb{R}^{n \times n}$. It is easy to see that $\beta \in \mathbb{M}_{d}^{-}$ is equivalent to the fact that $\beta_{I I} \in \mathbb{M}_{m}^{-}$and $\beta_{J J} \in \mathbb{M}_{n}^{-}$.

We now make a few comments on Theorem 2.6. To our knowledge, Theorem [2.6] seems to be the first result towards the existence of limiting distributions for affine processes on $D$ in such a generality. It includes many previous results as special cases. In particular, it covers [12, Theorem 2.4] for affine diffusions, and partially extends [23, Theorem 4.1] for OU-type processes and [22, 
Corollary 2] for 1-dimensional CBI processes. However, we are not able to show $\int_{\{\|\xi\|>1\}} \log \|\xi\| m(\mathrm{~d} \xi)<\infty$, provided that $\beta \in \mathbb{M}_{d}^{-}$and the stationarity of $X$ is known.

Our strategy of proving Theorem 2.6 is as follows. Clearly, to prove the weak convergence of the distribution of $X_{t}$ to $\pi$, it is essential to establish the pointwise convergence of the corresponding characteristic functions, i.e.,

$\mathbb{E}_{x}\left[\mathrm{e}^{\left\langle X_{t}, u\right\rangle}\right]=\exp \{\phi(t, u)+\langle x, \psi(t, u)\rangle\} \rightarrow \exp \left\{\int_{0}^{\infty} F(\psi(s, u)) \mathrm{d} s\right\} \quad$ as $t \rightarrow \infty$.

We will proceed in two steps. In the first step, we prove that for each $u \in \mathcal{U}, \psi(t, u)$ converges to zero exponentially fast. For $u$ in a small neighborhood of the origin, this convergence follows by a fine analysis of the generalized Riccati equations (2.5), (2.7) and an application of the linearized stability theorem for ODEs. Then, by some probabilistic arguments, we show that $\psi(t, u)$ reaches every neighborhood of the origin for large enough $t$. The essential observation here is the tightness of the laws of $X_{t}, t \geqslant 0$. This is a simple consequence of the uniform boundedness for the first moment of $X_{t}, t \geqslant 0$, which we show in Proposition 3.8. We thus obtain the desired convergence speed of $\psi(t, u) \rightarrow 0$ by the semi-flow property (2.3). In the second step, we show that

$$
\phi(t, u)=\int_{0}^{t} F(\psi(s, u)) \mathrm{d} s \rightarrow \int_{0}^{\infty} F(\psi(s, u)) \mathrm{d} s \quad \text { as } t \rightarrow \infty .
$$

Since $\psi(s, u) \rightarrow 0$ exponentially fast as $s \rightarrow \infty$, we will see that the convergence in (2.9) is naturally connected with the condition $\int_{\{\|\xi\|>1\}} \log \|\xi\| m(\mathrm{~d} \xi)<\infty$. Finally, the stationarity of $\pi$ can be derived using the semi-flow property.

\section{Large time behavior of the function $\psi(t, u)$}

In this section we consider an affine process $X$ with admissible parameters $(a, \alpha, b, \beta, m, \mu)$ and assume that

$$
a=0, b=0, m=0 .
$$

In particular, we have $F \equiv 0$ as well as $\phi \equiv 0$. We will show that if $\beta \in \mathbb{M}_{d}^{-}$, then $\psi(t, u) \rightarrow 0$ exponentially fast as $t \rightarrow \infty$.

Remark 3.1. The assumption that $a=0, b=0$ and $m=0$ is not essential. Indeed, Proposition 3.10, as the main result of this section, remains true if we drop Assumption (3.1). This follows from the following observation: when we study the properties of the function $\psi(t, u)$, the parameters $a, b$ and $m$ do not play a role.

3.1. Uniform boundedness for the first moment of $X_{t}, t \geqslant 0$. The aim we pursue in this subsection is to establish the uniform boundedness for the first moment of $X_{t}, t \geqslant 0$. We start with some approximations of $X$, which were introduced in 4 .

For $K \in(1, \infty)$, let

$$
\mu_{K, i}(\mathrm{~d} \xi):=\mathbb{1}_{\{\|\xi\| \leqslant K\}}(\xi) \mu_{i}(\mathrm{~d} \xi)
$$


and denote by $\left(X_{K, t}\right)_{t \geqslant 0}$ the affine process with admissible parameters ( $a=$ $\left.0, \alpha, b=0, \beta, m=0, \mu_{K}\right)$, where $\mu_{K}=\left(\mu_{K, 1}, \ldots, \mu_{K, m}\right)$. Then we have

$$
\mathbb{E}_{x}\left[\mathrm{e}^{\left\langle X_{K, t}, u\right\rangle}\right]=\exp \left\{\left\langle x, \psi_{K}(t, u)\right\rangle\right\}, \quad t \geqslant 0, x \in D, u \in \mathcal{U},
$$

for some function $\psi_{K}: \mathbb{R}_{\geqslant 0} \times \mathcal{U} \rightarrow \mathbb{C}^{d}$. By (2.5) and (2.6), we know that $\psi_{K}=$ $\left(\psi_{K}^{I}, \psi^{J}\right)$, where $\psi^{J}(t, u)=\exp \left(\beta_{J J}^{\top} t\right) w$ for $u=(v, w) \in \mathbb{C}_{\leqslant 0}^{m} \times \mathrm{i} \mathbb{R}^{n}$ and $\psi_{K}^{I}$ satisfies the generalized Riccati equation

$$
\partial_{t} \psi_{K}^{I}(t, u)=R_{K}^{I}\left(\psi_{K}^{I}(t, u), \mathrm{e}^{\beta_{J J}^{\top} t} w\right), \quad \psi_{K}^{I}(0, u)=v \in \mathbb{C}_{\leqslant 0}^{m},
$$

where $R_{K}^{I}=\left(R_{K, i}, \ldots, R_{K, m}\right)^{\top}$ with

$$
R_{K, i}(u)=\left\langle u, \alpha_{i} u\right\rangle+\sum_{k=1}^{d} \beta_{k i} u_{k}+\int_{D \backslash\{0\}}\left(\mathrm{e}^{\langle u, \xi\rangle}-1-\langle u, \xi\rangle\right) \mu_{K, i}(\mathrm{~d} \xi), \quad i \in I .
$$

Lemma 3.2. For each $t \in \mathbb{R}_{\geqslant 0}$ and $u \in \mathcal{U}, \psi_{K}(t, u)$ converges to $\psi(t, u)$ as $K \rightarrow \infty$.

Proof. Clearly, we only need to show the pointwise convergence of $\psi_{K}^{I}$ to $\psi^{I}$. Let $u=(v, w) \in \mathbb{C}_{\leqslant 0}^{m} \times \mathbb{i}^{n}$ and $T>0$ be fixed.

By the Riccati equations for $\psi^{I}$ and $\psi_{K}^{I}$, we get

$$
\psi^{I}(t, u)=v+\int_{0}^{t} R^{I}\left(\psi^{I}(s, u), \mathrm{e}^{\beta_{J J}^{\top} s} w\right) \mathrm{d} s, \quad t \geqslant 0,
$$

and

$$
\psi_{K}^{I}(t, u)=v+\int_{0}^{t} R_{K}^{I}\left(\psi_{K}^{I}(s, u), \mathrm{e}^{\beta_{J}^{\top} s} w\right) \mathrm{d} s, \quad t \geqslant 0 .
$$

In view of the formula (6.16) in the proof of [8, Propostion 6.1], we have

$$
\begin{aligned}
& \sup _{t \in[0, T]}\left\|\psi_{K}^{I}(t, u)\right\|^{2} \leqslant \sup _{t \in[0, T]}\left(\|v\|^{2}\right.\left.+c_{1} \int_{0}^{t}\left(1+\left\|\mathrm{e}^{\beta_{J J}^{\top} s} w\right\|^{2}\right) \mathrm{d} s\right) \\
& \times \exp \left\{c_{1} \int_{0}^{t}\left(1+\left\|\mathrm{e}^{\beta_{J J}^{\top} s} w\right\|^{2}\right) \mathrm{d} s\right\} \\
& \leqslant\left(\|v\|^{2}+c_{1} \int_{0}^{T}\left(1+\left\|\mathrm{e}^{\beta_{J J}^{\top} s} w\right\|^{2}\right) \mathrm{d} s\right) \\
& \times \exp \left\{c_{1} \int_{0}^{T}\left(1+\left\|\mathrm{e}^{\beta_{J J}^{\top} s} w\right\|^{2}\right) \mathrm{d} s\right\},
\end{aligned}
$$

for some positive constant $c_{1}$. Moreover, by checking carefully the proof of 8 , Propostion 6.1] and noting that $\mu_{K, i} \leqslant \mu_{i}$, we can actually choose $c_{1}$ in such a way that it depends only on the parameters $\alpha, \beta, \mu$. So $c_{1}$ is independent of $K$. Similarly, the same inequality holds for $\psi^{I}$ :

$$
\sup _{t \in[0, T]}\left\|\psi^{I}(t, u)\right\|^{2} \leqslant\left(\|v\|^{2}+c_{1} \int_{0}^{T}\left(1+\left\|\mathrm{e}^{\beta_{J J}^{\top} s} w\right\|^{2}\right) \mathrm{d} s\right)
$$




$$
\times \exp \left\{c_{1} \int_{0}^{T}\left(1+\left\|\mathrm{e}^{\beta_{J J}^{\top} s} w\right\|^{2}\right) \mathrm{d} s\right\} .
$$

According to Lemma 2.5 the mapping $u \mapsto R^{I}(u): \mathcal{U} \rightarrow \mathbb{C}^{m}$ is locally Lipschitz continuous. Therefore, for each $L>0$, there exists a constant $c_{2}=c_{2}(L)>0$ such that

$$
\left\|R_{i}\left(u_{1}\right)-R_{i}\left(u_{2}\right)\right\| \leqslant c_{2}\left\|u_{1}-u_{2}\right\|, \quad \text { for all } i \in I \text { and }\left\|u_{1}\right\|,\left\|u_{2}\right\| \leqslant L .
$$

In addition, it is easy to see that for $u \in \mathcal{U}$,

$$
\begin{aligned}
\left\|R_{i}(u)-R_{K, i}(u)\right\| & =\left|\int_{\{\|\xi\|>K\}}\left(\mathrm{e}^{\langle u, \xi\rangle}-1-\langle u, \xi\rangle\right) \mu_{i}(\mathrm{~d} \xi)\right| \\
& \leqslant \int_{\{\|\xi\|>K\}} 2 \mu_{i}(\mathrm{~d} \xi)+\|u\| \int_{\{\|\xi\|>K\}}\|\xi\| \mu_{i}(\mathrm{~d} \xi) \\
& \leqslant \varepsilon_{K}(1+\|u\|),
\end{aligned}
$$

where $\varepsilon_{K}:=\sum_{i=1}^{m} \int_{\{\|\xi\|>K\}}(2+\|\xi\|) \mu_{i}(\mathrm{~d} \xi)$. Note that $\varepsilon_{K} \rightarrow 0$ as $K \rightarrow \infty$ by dominated convergence.

Let

$$
g_{K}(t):=\left\|\psi^{I}(t, u)-\psi_{K}^{I}(t, u)\right\|, \quad t \in[0, T] .
$$

By (3.2) and (3.3), we have

$$
\begin{aligned}
g_{K}(t) \leqslant & \left\|\int_{0}^{t} R^{I}\left(\psi^{I}(s, u), \mathrm{e}^{\beta_{J J}^{\top} s} w\right) \mathrm{d} s-\int_{0}^{t} R_{K}^{I}\left(\psi_{K}^{I}(s, u), \mathrm{e}^{\beta_{J J}^{\top} s} w\right) \mathrm{d} s\right\| \\
\leqslant & \sum_{i=1}^{m} \int_{0}^{t}\left\|R_{i}\left(\psi^{I}(s, u), \mathrm{e}^{\beta_{J J}^{\top} s} w\right)-R_{i}\left(\psi_{K}^{I}(s, u), \mathrm{e}^{\beta_{J J}^{\top} s} w\right)\right\| \mathrm{d} s \\
& +\sum_{i=1}^{m} \int_{0}^{t}\left\|R_{i}\left(\psi_{K}^{I}(s, u), \mathrm{e}^{\beta_{J}^{\top} s} w\right)-R_{K, i}\left(\psi_{K}^{I}(s, u), \mathrm{e}^{\beta_{J J}^{\top} s} w\right)\right\| \mathrm{d} s .
\end{aligned}
$$

In virtue of (3.4), there exists a constant $c_{3}=c_{3}(T)>0$ such that

$$
\sup _{K \in[1, \infty)} \sup _{s \in[0, T]}\left\|\psi_{K}^{I}(s, u)\right\| \leqslant c_{3}<\infty
$$

which implies

$$
\sup _{K \in[1, \infty)} \sup _{s \in[0, T]}\left\|\left(\psi_{K}^{I}(s, u), \mathrm{e}^{\beta_{J J}^{\top} s} w\right)\right\| \leqslant c_{4}<\infty .
$$

So, for $0<s \leqslant T$, we get

$$
\left\|R_{i}\left(\psi^{I}(s, u), \mathrm{e}^{\beta_{J J}^{\top} s} w\right)-R_{i}\left(\psi_{K}^{I}(s, u), \mathrm{e}^{\beta_{J J}^{\top} s} w\right)\right\| \leqslant c_{5}\left\|\psi^{I}(s, u)-\psi_{K}^{I}(s, u)\right\|
$$

from (3.5), and obtain

$$
\left\|R_{i}\left(\psi_{K}^{I}(s, u), \mathrm{e}^{\beta_{J J}^{\top} s} w\right)-R_{K, i}\left(\psi_{K}^{I}(s, u), \mathrm{e}^{\beta_{J J}^{\top} s} w\right)\right\| \leqslant \varepsilon_{K}\left(1+c_{6}\right)
$$

from (3.6) and (3.8). Here, $c_{5}, c_{6}>0$ are constants not depending on $K$. 
Combining (3.7), (3.9) and (3.10) yields, for $t \in[0, T]$,

$$
\begin{aligned}
g_{K}(t) & \leqslant c_{5} m \int_{0}^{t}\left\|\psi^{I}(s, u)-\psi_{K}^{I}(s, u)\right\| \mathrm{d} s+m \varepsilon_{K}\left(1+c_{6}\right) t \\
& =c_{5} m \int_{0}^{t} g_{K}(s) \mathrm{d} s+m \varepsilon_{K}\left(1+c_{6}\right) t .
\end{aligned}
$$

Gronwall's inequality implies

$$
\begin{aligned}
g_{K}(t) & \leqslant m \varepsilon_{K}\left(1+c_{6}\right) t+m^{2} \varepsilon_{K}\left(1+c_{6}\right) c_{5} \int_{0}^{t} s \mathrm{e}^{c_{5} m(t-s)} \mathrm{d} s \\
& \leqslant m \varepsilon_{K}\left(1+c_{6}\right)\left(T+c_{5} m T^{2} \mathrm{e}^{c_{5} m T}\right), \quad t \in[0, T] .
\end{aligned}
$$

Since $\varepsilon_{K} \rightarrow 0$ as $K \rightarrow \infty$, we see that $g_{K}(t) \rightarrow 0$ and thus

$$
\psi_{K}^{I}(t, u) \rightarrow \psi^{I}(t, u), \quad \text { for all } t \in[0, T] .
$$

For $K \in(1, \infty)$, the generator $\mathcal{A}_{K}$ of $\left(X_{K, t}\right)_{t \geqslant 0}$ is given by

$$
\begin{aligned}
\mathcal{A}_{K} f(x)= & \sum_{k, l=1}^{d}\left(\sum_{i=1}^{m} \alpha_{i, k l} x_{i}\right) \frac{\partial^{2} f(x)}{\partial x_{k} \partial x_{l}}+\langle\beta x, \nabla f(x)\rangle \\
& +\sum_{i=1}^{m} x_{i} \int_{D \backslash\{0\}}(f(x+\xi)-f(x)-\langle\nabla f(x), \xi\rangle) \mu_{K, i}(\mathrm{~d} \xi),
\end{aligned}
$$

defined for every $f \in C_{c}^{2}(D)$.

To avoid the complication of discussing the domain of definition for the generator $\mathcal{A}_{K}$, we introduce the operator $\mathcal{A}_{K}^{\sharp}$, which was also used in $[8]$.

Definition 3.3. If $f \in C^{2}(D)$ is such that for all $x \in D$,

$$
\sum_{i=1}^{m} \int_{D \backslash\{0\}}|f(x+\xi)-f(x)-\langle\nabla f(x), \xi\rangle| \mu_{K, i}(\mathrm{~d} \xi)<\infty,
$$

then we say that $\mathcal{A}_{K}^{\sharp} f$ is well-defined and let

$$
\begin{aligned}
\mathcal{A}_{K}^{\sharp} f(x):= & \sum_{k, l=1}^{d}\left(\sum_{i=1}^{m} \alpha_{i, k l} x_{i}\right) \frac{\partial^{2} f(x)}{\partial x_{k} \partial x_{l}}+\langle\beta x, \nabla f(x)\rangle \\
& +\sum_{i=1}^{m} x_{i} \int_{D \backslash\{0\}}(f(x+\xi)-f(x)-\langle\nabla f(x), \xi\rangle) \mu_{K, i}(\mathrm{~d} \xi)
\end{aligned}
$$

for $x \in D$.

It is easy to see that if $f \in C^{2}(D)$ has bounded first and second order derivatives, then $\mathcal{A}_{K}^{\sharp} f$ is well-defined.

Recall that the matrix $\beta$ can be written as in (2.8). We define the following matrices

$$
M_{1}:=\int_{0}^{\infty} \mathrm{e}^{t \beta_{I I}^{\top}} \mathrm{e}^{t \beta_{I I}} \mathrm{~d} t \quad \text { and } \quad M_{2}:=\int_{0}^{\infty} \mathrm{e}^{t \beta_{J J}^{\top}} \mathrm{e}^{t \beta_{J J}} \mathrm{~d} t .
$$


Since $\beta_{I I} \in \mathbb{M}_{m}^{-}$and $\beta_{J J} \in \mathbb{M}_{n}^{-}$, the matrices $M_{1}$ and $M_{2}$ are well-defined. Moreover, we have that $M_{1} \in \mathbb{S}_{m}^{++}$and $M_{2} \in \mathbb{S}_{n}^{++}$. In the following we will often write $x=(y, z) \in \mathbb{R}_{\geqslant 0}^{m} \times \mathbb{R}^{n}$ for $x \in D$. For $y_{1}, y_{2} \in \mathbb{R}_{\geqslant 0}^{m}$ and $z_{1}, z_{2} \in \mathbb{R}^{n}$, we define

$$
\left\langle y_{1}, y_{2}\right\rangle_{I}:=\int_{0}^{\infty}\left\langle\mathrm{e}^{t \beta_{I I}} y_{1}, \mathrm{e}^{t \beta_{I I}} y_{2}\right\rangle \mathrm{d} t \quad \text { and }\left\langle z_{1}, z_{2}\right\rangle_{J}:=\int_{0}^{\infty}\left\langle\mathrm{e}^{t \beta_{J J}} z_{1}, \mathrm{e}^{t \beta_{J J}} z_{2}\right\rangle \mathrm{d} t .
$$

It is easily verified that $\langle\cdot, \cdot\rangle_{I}$ and $\langle\cdot, \cdot\rangle_{J}$ define inner products on $\mathbb{R}^{m}$ and $\mathbb{R}^{n}$, respectively. Moreover, we have that

$$
\left\langle y_{1}, y_{2}\right\rangle_{I}=y_{2}^{\top} M_{1} y_{1}=\left\langle y_{1}, M_{1} y_{2}\right\rangle \quad \text { and } \quad\left\langle z_{1}, z_{2}\right\rangle_{J}=z_{2}^{\top} M_{2} z_{1}=\left\langle z_{1}, M_{2} z_{2}\right\rangle .
$$

The norms on $\mathbb{R}^{m}$ and $\mathbb{R}^{n}$ induced by the scalar products $\langle\cdot, \cdot\rangle_{I}$ and $\langle\cdot, \cdot\rangle_{J}$ are denoted by

$$
\|y\|_{I}:=\sqrt{\langle y, y\rangle_{I}} \text { and }\|z\|_{J}:=\sqrt{\langle z, z\rangle_{J}}
$$

respectively.

In the following lemma we construct a Lyapunov function $V$ for $\left(X_{K, t}\right)_{t \geqslant 0}$. Note that the definition of $V$ does not depend on $K$.

Lemma 3.4. Assume $m \geqslant 1$ and $n \geqslant 1$. Suppose that $\beta \in \mathbb{M}_{d}^{-}$. Let $V \in C^{2}(D, \mathbb{R})$ be such that $V>0$ on $D$ and

$$
V(x)=\left(\langle y, y\rangle_{I}+\varepsilon\langle z, z\rangle_{J}\right)^{1 / 2}, \quad \text { whenever } x=(y, z) \in \mathbb{R}_{\geqslant 0}^{m} \times \mathbb{R}^{n} \text { with }\|x\|>2 .
$$

Here $\varepsilon>0$ is some small enough constant. Then $\mathcal{A}_{K}^{\sharp} V$ is well-defined and $V$ is a Lyapunov function for $\left(X_{K, t}\right)_{t \geqslant 0}$, that is, there exist positive constants $c$ and $C$ such that

$$
\mathcal{A}_{K}^{\sharp} V(x) \leqslant-c V(x)+C, \quad \text { for all } x \in D .
$$

Moreover, the constants $c$ and $C$ can be chosen to be independent of $K$.

Proof. For $x_{1}=\left(y_{1}, z_{1}\right) \in \mathbb{R}_{\geqslant 0}^{m} \times \mathbb{R}^{n}$ and $x_{2}=\left(y_{2}, z_{2}\right) \in \mathbb{R}_{\geqslant 0}^{m} \times \mathbb{R}^{n}$, we define

$$
\left\langle x_{1}, x_{2}\right\rangle_{\beta}:=\left\langle y_{1}, z_{1}\right\rangle_{I}+\varepsilon\left\langle y_{2}, z_{2}\right\rangle_{J},
$$

where $\varepsilon>0$ is a small constant to be determined later. Set $\tilde{V}(x):=\left(\langle x, x\rangle_{\beta}\right)^{1 / 2}$, $x \in D$. Then $\widetilde{V}$ is smooth on $\{x \in D:\|x\|>1\}$. By the extension lemma for smooth functions (see [19, Lemma 2.26]), we can easily find a function $V \in$ $C^{\infty}(D, \mathbb{R})$ such that $V>0$ on $D$ and $V(x)=\widetilde{V}(x)=\left(\langle x, x\rangle_{\beta}\right)^{1 / 2}$ for $\|x\|>2$. So for all $x=(y, z) \in \mathbb{R}_{\geqslant 0}^{m} \times \mathbb{R}^{n}$ with $\|x\|>2$, we have

$$
\nabla V(y, z)=V(y, z)^{-1}\left(\begin{array}{c}
M_{1} y \\
\varepsilon M_{2} z
\end{array}\right)
$$

and

$$
\nabla^{2} V(y, z)=\left(\begin{array}{cc}
\frac{M_{1}}{V(y, z)}-\frac{\left(M_{1} y\right)\left(M_{1} y\right)^{\top}}{V(y, z)^{3}} & \frac{-\varepsilon\left(M_{1} y\right)\left(M_{2} z\right)^{\top}}{V(y, z)^{3}} \\
\frac{-\varepsilon\left(M_{1} y\right)\left(M_{2} z\right)^{\top}}{V(y, z)^{3}} & \frac{\varepsilon M_{2}}{V(y, z)}-\frac{\varepsilon^{2}\left(M_{2} z\right)\left(M_{2} z\right)^{\top}}{V(y, z)^{3}}
\end{array}\right)
$$


PENG JIN, JONAS KREMER, AND BARBARA RÜDIGER

We write $\mathcal{A}_{K}^{\sharp} V=\mathcal{D} V+\mathcal{J}_{K} V$, where

$$
\begin{aligned}
\mathcal{D} V(x) & :=\sum_{k, l=1}^{d}\left\langle\alpha_{I, k l}, x_{I}\right\rangle \frac{\partial^{2} V(x)}{\partial x_{k} \partial x_{l}}+\langle\beta x, \nabla V(x)\rangle, \\
\mathcal{J}_{K} V(x) & :=\sum_{i=1}^{m} x_{i} \int_{D \backslash\{0\}}(V(x+\xi)-V(x)-\langle\nabla V(x), \xi\rangle) \mu_{K, i}(\mathrm{~d} \xi) .
\end{aligned}
$$

We now estimate $\mathcal{D} V(x)$ and $\mathcal{J}_{K} V(x)$ separately. Let us first consider $\mathcal{D} V(x)$. We may further split $\mathcal{D} V(x)$ into the drift part and the diffusion part.

Drift. Recall that $\beta_{I J}=0$. Consider $x=(y, z)$ with $\|x\|>2$. It follows from (3.11) that

$$
\begin{aligned}
\langle\beta x, \nabla V(x)\rangle & =\left\langle\left(\begin{array}{c}
\beta_{I I} y \\
\beta_{J I} y+\beta_{J J} z
\end{array}\right),\left(\begin{array}{c}
V(y, z)^{-1} M_{1} y \\
V(y, z)^{-1} \varepsilon M_{2} z
\end{array}\right)\right\rangle \\
& =V(y, z)^{-1}\left(\left\langle\beta_{I I} y, M_{1} y\right\rangle+\left\langle\beta_{J I} y, \varepsilon M_{2} z\right\rangle+\left\langle\beta_{J J} z, \varepsilon M_{2} z\right\rangle\right) .
\end{aligned}
$$

The first and the third inner product on the right-hand side may be estimated similarly. Namely, we have

$$
V(y, z)^{-1}\left\langle\beta_{I I} y, M_{1} y\right\rangle=\frac{1}{2} V(y, z)^{-1} y^{\top}\left(M_{1} \beta_{I I}+\beta_{I I}^{\top} M_{1}\right) y .
$$

The definition of $M_{1}$ implies

$$
\begin{aligned}
M_{1} \beta_{I I}+\beta_{I I}^{\top} M_{1} & =\int_{0}^{\infty}\left(\mathrm{e}^{t \beta_{I I}^{\top}} \mathrm{e}^{t \beta_{I I}} \beta_{I I}+\beta_{I I}^{\top} \mathrm{e}^{t \beta_{I I}^{\top}} \mathrm{e}^{t \beta_{I I}}\right) \mathrm{d} t \\
& =\int_{0}^{\infty}\left(\frac{\mathrm{d}}{\mathrm{d} t} \mathrm{e}^{t \beta_{I I}^{\top}} \mathrm{e}^{t \beta_{I I}}\right) \mathrm{d} t \\
& =\left.\mathrm{e}^{t \beta_{I I}^{\top}} \mathrm{e}^{t \beta_{I I}}\right|_{t=0} ^{\infty} \\
& =-I_{m},
\end{aligned}
$$

where $I_{m}$ denotes the $m \times m$ identity matrix. Hence

$$
V(y, z)^{-1}\left\langle\beta_{I I} y, M_{1} y\right\rangle=-\frac{1}{2} V(y, z)^{-1} y^{\top} y .
$$

Since all norms on $\mathbb{R}^{m}$ are equivalent, we have

$$
-y^{\top} y \leqslant-c_{1} y^{\top} M_{1} y=-c_{1}\langle y, y\rangle_{I} \leqslant-c_{1}\|y\|_{I}^{2},
$$

for some positive constant $c_{1}$ that is independent of $K$. So

$$
V(y, z)^{-1}\left\langle\beta_{I I} y, M_{1} y\right\rangle \leqslant-c_{1}\|y\|_{I}^{2} V(y, z)^{-1} .
$$

In the very same way we obtain

$$
V(y, z)^{-1}\left\langle\beta_{J} z, \varepsilon M_{2} z\right\rangle \leqslant-c_{2} \varepsilon\|z\|_{J}^{2} V(y, z)^{-1},
$$

for some constant $c_{2}>0$. To estimate the remaining term, we can use Cauchy Schwarz inequality to obtain

$$
\begin{aligned}
\left|V(y, z)^{-1}\left\langle\beta_{J I} y, \varepsilon M_{2} z\right\rangle\right| & \leqslant \varepsilon V(y, z)^{-1}\left\|\beta_{J I} y\right\|\left\|M_{2} z\right\| \\
& \leqslant c_{3} \varepsilon V(y, z)^{-1}\|y\|\|z\|,
\end{aligned}
$$


for some constant $c_{3}>0$. Using the fact that all norms on $\mathbb{R}^{d}$ are equivalent, we get

$$
\begin{aligned}
\left|V(y, z)^{-1}\left\langle\beta_{J I} y, \varepsilon M_{2} z\right\rangle\right| & \leqslant \varepsilon c_{4} V(y, z)^{-1}\|y\|_{I}\|z\|_{J} \\
& =c_{4} \frac{\sqrt{\varepsilon} \sqrt{\langle y, y\rangle_{I}} \sqrt{\varepsilon\langle z, z\rangle_{J}}}{\sqrt{\langle y, y\rangle_{I}+\varepsilon\langle z, z\rangle_{J}}} \\
& \leqslant c_{4} \sqrt{\varepsilon}\|y\|_{I} .
\end{aligned}
$$

Combining (3.15), (3.16) and (3.17), we obtain

$$
\begin{aligned}
\langle\beta x, \nabla V(x)\rangle & \leqslant-c_{1}\|y\|_{I}^{2} V(y, z)^{-1}-\varepsilon c_{2}\|z\|_{J}^{2} V(y, z)^{-1}+c_{4} \sqrt{\varepsilon}\|y\|_{I} \\
& \leqslant-c_{5}\left(\langle y, y\rangle_{I}+\varepsilon\langle z, z\rangle_{J}\right) V(y, z)^{-1}+c_{4} \sqrt{\varepsilon}\|y\|_{I} \\
& \leqslant-c_{5} V(y, z)+c_{4} \sqrt{\varepsilon} V(y, z),
\end{aligned}
$$

where $c_{5}:=c_{1} \wedge c_{2}>0$. Since $c_{4}$ and $c_{5}$ depend only on $\beta$ but not on $\varepsilon$, by choosing $\varepsilon=\varepsilon_{0}>0$ sufficiently small, we get

$$
\langle\beta x, \nabla V(x)\rangle \leqslant-c_{6} V(x), \quad x \in D \quad \text { with }\|x\|>2 .
$$

From now on we take $\varepsilon=\varepsilon_{0}$ as fixed. In particular, the upcoming constants $c_{7}-c_{11}$ may depend on $\varepsilon$.

Diffusion. By (3.12), we have

$$
\left|\frac{\partial^{2} V(x)}{\partial x_{k} \partial x_{l}}\right| \leqslant \frac{c_{7}}{V(x)}, \quad \text { for all }\|x\|>2, k, l \in\{1, \ldots, d\},
$$

where $c_{7}>0$ is a constant. This implies

$$
\sup _{x \in D}\left|x_{i} \frac{\partial^{2} V(x)}{\partial x_{k} \partial x_{l}}\right|<\infty, \quad \text { for all } i \in I \text { and } k, l \in\{1, \ldots, d\} .
$$

We conclude that

$$
\left|\sum_{k, l=1}^{d}\left(\sum_{i \in I} \alpha_{i, k l} x_{i}\right) \frac{\partial^{2} V(x)}{\partial x_{k} \partial x_{l}}\right| \leqslant c_{8}, \quad \text { for all } x \in D,
$$

where $c_{8}>0$ is a constant.

Turning to the jump part $\mathcal{J}_{K}$, we define for $i \in I$ and $k \in \mathbb{N}$,

$$
\mathcal{J}_{k, i, *} V(x):=x_{i} \int_{\{0<\|\xi\|<k\}}(V(x+\xi)-V(x)-\langle\nabla V(x), \xi\rangle) \mu_{K, i}(\mathrm{~d} \xi),
$$

and

$$
\mathcal{J}_{k, i}^{*} V(x):=x_{i} \int_{\{\|\xi\| \geqslant k\}}(V(x+\xi)-V(x)-\langle\nabla V(x), \xi\rangle) \mu_{K, i}(\mathrm{~d} \xi) .
$$

So $\mathcal{J}_{K} V(x)=\sum_{i \in I}\left(\mathcal{J}_{k, i, *} V(x)+\mathcal{J}_{k, i}^{*} V(x)\right)$.

Big jumps. By the mean value theorem, we get

$$
\begin{aligned}
\left|\mathcal{J}_{k, i}^{*} V(x)\right| & \leqslant\left\|x_{i}\right\| \int_{\{\|\xi\| \geqslant k\}}\left(\|\nabla V\|_{\infty}\|\xi\|+\|\nabla V(x)\|\|\xi\|\right) \mu_{i}(\mathrm{~d} \xi) \\
& \leqslant 2\|x\|\|\nabla V\|_{\infty} \int_{\{\|\xi\| \geqslant k\}}\|\xi\| \mu_{i}(\mathrm{~d} \xi)
\end{aligned}
$$




$$
\leqslant c_{9}(1+V(x)) \int_{\{\|\xi\| \geqslant k\}}\|\xi\| \mu_{i}(\mathrm{~d} \xi)<\infty,
$$

where we used that $\|\nabla V\|_{\infty}=\sup _{x \in D}\|\nabla V(x)\|<\infty$, as a consequence of (3.11). Hence, by dominated convergence, we can find large enough $k=k_{0}>0$ such that

$$
\left|\mathcal{J}_{k_{0}, i}^{*} V(x)\right| \leqslant \frac{1}{2} c_{6}(1+V(x)), \quad x \in D .
$$

Small jumps. To estimate the small jump part, we apply (3.19) and the mean value theorem, yielding for $\|x\|>3 k_{0}$,

$$
\begin{aligned}
\left|\mathcal{J}_{k_{0}, i, *} V(x)\right| & \leqslant\left|x_{i} \int_{\left\{0<\|\xi\|<k_{0}\right\}}\left(\int_{0}^{1}\langle\nabla V(x+r \xi)-\nabla V(x), \xi\rangle\right) \mathrm{d} r \mu_{K, i}(\mathrm{~d} \xi)\right| \\
& \leqslant\left\|x_{i}\right\| \sup _{\widetilde{x} \in B_{k_{0}}(x)}\left\|\nabla^{2} V(\widetilde{x})\right\| \int_{\left\{0<\|\xi\|<k_{0}\right\}}\|\xi\|^{2} \mu_{i}(\mathrm{~d} \xi) \\
& \leqslant c_{7}\|x\| \sup _{\widetilde{x} \in B_{k_{0}}(x)} \frac{1}{V(\widetilde{x})} \int_{\left\{0<\|\xi\|<k_{0}\right\}}\|\xi\|^{2} \mu_{i}(\mathrm{~d} \xi) \\
& \leqslant c_{10} \frac{\|x\|}{\|x\|-k_{0}} \leqslant 2 c_{10}<\infty,
\end{aligned}
$$

with some positive constant $c_{10}$ not depending on $K$. Here $B_{k_{0}}(x)$ denotes the ball with center $x$ and radius $k_{0}$. Note that $\mathcal{J}_{k_{0}, i, *} V(x)$ is continuous in $x \in D$. Hence, we conclude that

$$
\left|\mathcal{J}_{K} V(x)\right| \leqslant \frac{1}{2} c_{6} V(x)+c_{11}, \quad x \in D .
$$

Combining the latter inequality with (3.18) and (3.20), we obtain the desired result, namely,

$$
\mathcal{A}_{K}^{\sharp} V(x)=\mathcal{D} V(x)+\mathcal{J}_{K} V(x) \leqslant-\frac{1}{2} c_{6} V(x)+c_{12}, \quad x \in D .
$$

Remark 3.5. For the function $V$ defined in the last lemma, we can easily find positive constants $c_{1}, c_{2}, c_{3}, c_{4}$ such that for all $x \in D$,

$$
V(x) \leqslant c_{1}\|x\|+c_{2} \quad \text { and } \quad\|x\| \leqslant c_{3} V(x)+c_{4} .
$$

Proposition 3.6. Assume $m \geqslant 1$ and $n \geqslant 1$. Suppose that $\beta \in \mathbb{M}_{d}^{-}$. Let $c, C$ and $V$ be the same as in Lemma 3.4. Then

$$
\mathbb{E}_{x}\left[V\left(X_{K, t}\right)\right] \leqslant \mathrm{e}^{-c t} V(x)+c^{-1} C \quad \text { for all } K \geqslant 1, x \in D \text { and } t \in \mathbb{R}_{\geqslant 0} .
$$

Proof. Let $x \in D, K \geqslant 1$ and $T>0$ be fixed. The proof is divided into three steps.

Step 1: We show that

$$
\sup _{t \in[0, T]} \mathbb{E}_{x}\left[\left\|X_{K, t}\right\|^{2}\right]<\infty
$$


Since $\mu_{K, i}$ has compact support, it follows that $\int_{\{\|\xi\|>1\}}\|\xi\|^{k} \mu_{K, i}(\mathrm{~d} \xi)<\infty$ for all $k \in \mathbb{N}$. By [8. Lemmas 5.3 and 6.5], we know that $\psi_{K} \in C^{2}\left(\mathbb{R}_{+} \times \mathcal{U}\right)$. Moreover, by [8, Theorem 2.16], we have

$$
\mathbb{E}_{x}\left[\left\|X_{K, t}\right\|^{2}\right]=-\sum_{l=1}^{d}\left(\left\langle x,\left.\partial_{\lambda_{l}}^{2} \psi_{K}(t, \mathrm{i} \lambda)\right|_{\lambda=0}\right\rangle+\left\langle x,\left.\partial_{\lambda_{l}} \psi_{K}(t, \mathrm{i} \lambda)\right|_{\lambda=0}\right\rangle^{2}\right),
$$

where the right-hand side is a continuous function in $t \in[0, T]$. So [3.25) follows.

Step 2: We show that

$$
\sup _{t \in[0, T]} \mathbb{E}_{x}\left[V\left(X_{K, t}\right)\right]<\infty .
$$

In fact, 3.26 follows from (3.23) and 3.25).

Step 3: We show that (3.24) is true. It follows from [8, Theorem 2.12] and [8, Lemma 10.1] that

$$
f\left(X_{K, t}\right)-f\left(X_{K, 0}\right)-\int_{0}^{t} \mathcal{A}_{K} f\left(X_{K, s}\right) \mathrm{d} s, \quad t \in \mathbb{R}_{\geqslant 0},
$$

is a $\mathbb{P}_{x}$-martingale for every $f \in C_{c}^{2}(D)$. Note that $V$ belongs to $C^{2}(D)$ but does not have compact support. Let $\varphi \in C_{c}^{\infty}\left(\mathbb{R}_{\geqslant 0}\right)$ be such that $\mathbb{1}_{[0,1]} \leqslant \varphi \leqslant \mathbb{1}_{[0,2]}$, and define $\left(\varphi_{j}\right)_{j \geqslant 1} \subset C_{c}^{\infty}(D)$ by $\varphi_{j}(y):=\varphi\left(\|y\|^{2} / j^{2}\right)$. Then

$$
\varphi_{j}(y)=1 \quad \text { for }\|y\| \leqslant j \quad \text { and } \quad \varphi_{j}(y)=0 \text { for }\|y\|>\sqrt{2} j,
$$

and $\varphi_{j} \rightarrow 1$ as $j \rightarrow \infty$. For $j \in \mathbb{N}$, we then define

$$
V_{j}(y):=V(y) \varphi_{j}(y), \quad y \in D .
$$

So $V_{j} \in C_{c}^{2}(D)$. In view of (3.27) and [10, Chap.4, Lemma 3.2], it follows that $\mathrm{e}^{c t} V_{j}\left(X_{K, t}\right)-V_{j}\left(X_{K, 0}\right)-\int_{0}^{t} \mathrm{e}^{c s} \mathcal{A}_{K} V_{j}\left(X_{K, s}\right) \mathrm{d} s-\int_{0}^{t} c \mathrm{e}^{c s} V_{j}\left(X_{K, s}\right) \mathrm{d} s, \quad t \in \mathbb{R}_{\geqslant 0}$, is a $\mathbb{P}_{x}$-martingale, and hence

$$
\mathrm{e}^{c t} \mathbb{E}_{x}\left[V_{j}\left(X_{K, t}\right)\right]-V_{j}(x)=\mathbb{E}_{x}\left[\int_{0}^{t} \mathrm{e}^{c s}\left(\mathcal{A}_{K} V_{j}\left(X_{K, s}\right)+c V_{j}\left(X_{K, s}\right)\right) \mathrm{d} s\right] .
$$

Now, a simple calculation shows

$$
\left\|\nabla \varphi_{j}(y)\right\| \leqslant \frac{2\|y\|}{j^{2}}\left\|\varphi^{\prime}\right\|_{\infty} \leqslant \frac{2 c_{1}\|y\|}{j^{2}}
$$

for some constant $c_{1}>0$. Therefore, by (3.23), we get

$$
\begin{aligned}
\left\|\nabla V_{j}(y)\right\| & =\mathbb{1}_{\{\|y\| \leqslant \sqrt{2} j\}}\left\|\varphi_{j}(y) \nabla V(y)+V(y) \nabla \varphi_{j}(y)\right\| \\
& \leqslant \mathbb{1}_{\{\|y\| \leqslant \sqrt{2} j\}}\left(\|\nabla V\|_{\infty}+c_{2}(1+\|y\|) \frac{2 c_{1}\|y\|}{j^{2}}\right) \\
& \leqslant c_{3} \frac{(1+j) j}{j^{2}}
\end{aligned}
$$


where $c_{2}$ and $c_{3}$ are positive constants. A similar calculation yields that there exists a constant $c_{4}>0$ such that

So

$$
\left\|\nabla^{2} \varphi_{j}(y)\right\| \leqslant c_{4} \frac{\|y\|^{2}+j^{2}}{j^{4}} .
$$

$$
\begin{aligned}
\left\|\nabla^{2} V_{j}(y)\right\| & \leqslant \mathbb{1}_{\{\|y\| \leqslant \sqrt{2} j\}}\left(\left\|\nabla^{2} V\right\|_{\infty}+2\|\nabla V\|_{\infty}\left\|\nabla \varphi_{j}(y)\right\|+\|V(y)\|\left\|\nabla^{2} \varphi_{j}(y)\right\|\right) \\
& \leqslant \mathbb{1}_{\{\|y\| \leqslant \sqrt{2} j\}}\left(c_{5}+\frac{c_{6}\|y\|}{j^{2}}+c_{7}(1+\|y\|) \frac{\|y\|^{2}+j^{2}}{j^{4}}\right) \\
& \leqslant c_{8} \frac{1+j+j^{2}}{j^{2}}
\end{aligned}
$$

where $c_{5}, c_{6}, c_{7}, c_{8}>0$ are constants. Define $\mathcal{D} V_{j}$ and $\mathcal{J}_{K} V_{j}$ similarly as in (3.13) and (3.14), respectively. It holds obviously that

$$
\left|\mathcal{D} V_{j}(y)\right| \leqslant c_{9}\|y\|\left(\left\|\nabla V_{j}\right\|_{\infty}+\left\|\nabla^{2} V_{j}\right\|_{\infty}\right), \quad y \in D .
$$

Similarly as in (3.21) and (3.22), we have that for all $y \in D$,

$$
\begin{aligned}
\left|\mathcal{J}_{K} V_{j}(y)\right| \leqslant c_{10} \| & y \| \sum_{i=1}^{m}\left(\left\|\nabla V_{j}\right\|_{\infty} \int_{\{\|\xi\| \geqslant 1\}}\|\xi\| \mu_{i}(\mathrm{~d} \xi)\right. \\
& \left.+\left\|\nabla^{2} V_{j}\right\|_{\infty} \int_{\{0<\|\xi\|<1\}}\|\xi\|^{2} \mu_{i}(\mathrm{~d} \xi)\right) .
\end{aligned}
$$

Using (3.28), (3.29) and the above estimates for $\mathcal{D} V_{j}$ and $\mathcal{J}_{K} V_{j}$, we obtain

$$
\left|\mathcal{A}_{K} V_{j}(y)\right| \leqslant c_{11}(1+\|y\|), \quad y \in D,
$$

where $c_{11}>0$ is a constant not depending on $j$. The dominated convergence theorem implies $\lim _{j \rightarrow \infty} \mathcal{A}_{K} V_{j}(y)=\mathcal{A}_{K}^{\sharp} V(y)$ for all $y \in D$. By (3.26), (3.30) and again dominated convergence, it follows that

$$
\mathrm{e}^{c t} \mathbb{E}_{x}\left[V\left(X_{K, t}\right)\right]-V(x)=\mathbb{E}_{x}\left[\int_{0}^{t} \mathrm{e}^{c s}\left(\mathcal{A}_{K}^{\sharp} V\left(X_{K, s}\right)+c V\left(X_{K, s}\right)\right) \mathrm{d} s\right] .
$$

Applying Lemma 3.4 yields

$$
\mathrm{e}^{c t} \mathbb{E}_{x}\left[V\left(X_{K, t}\right)\right]-V(x) \leqslant \mathbb{E}_{x}\left[\int_{0}^{t} \mathrm{e}^{c s} C \mathrm{~d} s\right] \leqslant c^{-1} C \mathrm{e}^{c t},
$$

which implies

$$
\mathbb{E}_{x}\left[V\left(X_{K, t}\right)\right] \leqslant \mathrm{e}^{-c t} V(x)+c^{-1}, \quad \text { fort } \in[0, T] .
$$

Since $x \in D, K \geqslant 1$ and $T>0$ are arbitrary, the assertion follows.

Arguing similarly as in Lemma 3.4 and Proposition 3.6 we obtain also an analog result for the case where $m \geqslant 1$ and $n=0$.

Proposition 3.7. Assume $m \geqslant 1$ and $n=0$. Suppose that $\beta \in \mathbb{M}_{d}^{-}$. Let $V \in$ $C^{2}(D, \mathbb{R})$ be such that $V>0$ on $D$ and

$$
V(x)=\langle x, x\rangle_{I}^{1 / 2}, \quad \text { whenever }\|x\|>2 .
$$


Then $\mathcal{A}_{K}^{\sharp} V$ is well-defined and there exist positive constants $c$ and $C$, independent of $K$, such that

$$
\mathcal{A}_{K}^{\sharp} V(x) \leqslant-c V(x)+C, \quad \forall x \in D .
$$

Moreover, for all $K \geqslant 1, t \geqslant 0$ and $x \in D$, it holds that

$$
\mathbb{E}_{x}\left[V\left(X_{K, t}\right)\right] \leqslant \mathrm{e}^{-c t} V(x)+c^{-1} C .
$$

We are now ready to prove the uniform boundedness for the first moment of $X_{t}, t \geqslant 0$.

Proposition 3.8. Let $X$ be an affine process satisfying (3.1). Suppose that $\beta \in$ $\mathbb{M}_{d}^{-}$. Then

$$
\sup _{t \geqslant 0} \mathbb{E}_{x}\left[\left\|X_{t}\right\|\right]<\infty \quad \text { for all } x \in D .
$$

Proof. If $m=0$ and $n \geqslant 1$, then $\left(X_{t}\right)_{t \geqslant 0}$ degenerates to a deterministic motion governed by the vector field $x \mapsto \beta x$. In this case we have

$$
X_{t}=\mathrm{e}^{\beta t} X_{0},
$$

so (3.31) follows from the assumption that $\beta \in \mathbb{M}_{d}^{-}$.

For the case where $m \geqslant 1$, by Propositions 3.6 and 3.7. we have

$$
\mathbb{E}_{x}\left[V\left(X_{K, t}\right)\right] \leqslant \mathrm{e}^{-c t} V(x)+c^{-1} C, \quad \text { for all } K \geqslant 1, x \in D \text { and } t \in \mathbb{R}_{\geqslant 0},
$$

where $c, C>0$ are constants not depending on $K$.

Let $x \in D$ be fixed and assume without loss of generality that $X_{0}=x$ a.s. In view of Lemma 3.2 and Skorokhod's representation theorem (see, e.g., 10, Chap.3, Theorem 1.8]), there exist some probability space $(\widetilde{\Omega}, \widetilde{\mathcal{F}}, \widetilde{\mathbb{P}})$ on which $\left(\widetilde{X}_{K, t}\right)_{K \geqslant 1}$ and $\widetilde{X}_{t}$ are defined such that $\widetilde{X}_{K, t}$ and $\widetilde{X}_{t}$ have the same distributions as $X_{K, t}$ and

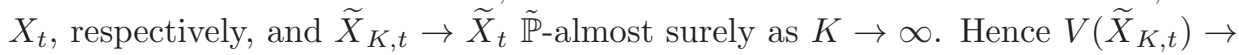
$V\left(\widetilde{X}_{t}\right) \tilde{\mathbb{P}}$-almost surely as $K \rightarrow \infty$. By (3.32) and Fatou's lemma, we have

$$
\begin{aligned}
\mathbb{E}_{x}\left[V\left(X_{t}\right)\right]=\widetilde{\mathbb{E}}\left[V\left(\widetilde{X}_{t}\right)\right] & \leqslant \liminf _{K \rightarrow \infty} \widetilde{\mathbb{E}}\left[V\left(\widetilde{X}_{K, t}\right)\right] \\
& =\liminf _{K \rightarrow \infty} \mathbb{E}_{x}\left[V\left(X_{K, t}\right)\right] \\
& \leqslant \mathrm{e}^{-c t} V(x)+c^{-1} C
\end{aligned}
$$

for all $t \geqslant 0$. By (3.23), the assertion follows.

3.2. Exponential convergence of $\psi(t, u)$ to zero. In this subsection we study the convergence speed of $\psi(t, u) \rightarrow 0$ as $t \rightarrow \infty$.

Lemma 3.9. Suppose that $\beta \in \mathbb{M}_{d}^{-}$. There exist $\delta>0$ and constants $C_{1}, C_{2}>0$ such that for all $u \in \mathcal{U}$ with $\|u\|<\delta$,

$$
\|\psi(t, u)\| \leqslant C_{1} \exp \left\{-C_{2} t\right\}, \quad t \geqslant 0 .
$$

Proof. For $u \in \mathcal{U}$, we can write $u=(v, w) \in \mathbb{C}_{\leqslant 0}^{m} \times \mathrm{i} \mathbb{R}^{n}$ and further $v=x+\mathrm{i} y$ and $w=\mathrm{i} z$, where $x \in \mathbb{R}_{\leqslant 0}^{m}, y \in \mathbb{R}^{m}$ and $z \in \mathbb{R}^{n}$. Therefore,

$$
\psi(t, u)=\psi(t, v, w)=\left(\begin{array}{c}
\psi^{I}(t, x+\mathrm{i} y, \mathrm{i} z) \\
\mathrm{ie}^{\beta_{J J}^{\top} t} z
\end{array}\right) .
$$


For $x \in \mathbb{R}_{\leqslant 0}^{m}, y \in \mathbb{R}^{m}$, and $z \in \mathbb{R}^{n}$, we define

$$
\widetilde{\psi}(t, x, y, z):=\left(\begin{array}{c}
\operatorname{Re} \psi^{I}(t, x+\mathrm{i} y, \mathrm{i} z) \\
\operatorname{Im} \psi^{I}(t, x+\mathrm{i} y, \mathrm{i} z) \\
\mathrm{e}^{\beta_{J J}^{\top} t} z
\end{array}\right)=\left(\begin{array}{l}
\vartheta \\
\eta \\
\zeta
\end{array}\right), \quad t \geqslant 0 .
$$

Recall that $\psi^{I}(t, u)$ satisfies the Riccati equation

$$
\partial_{t} \psi^{I}(t, v, w)=R^{I}\left(\psi^{I}(t, v, w), \mathrm{e}^{\beta_{J J}^{\top} t} w\right), \quad \psi^{I}(0, v, w)=v .
$$

So

$$
\begin{aligned}
& \partial_{t} \widetilde{\psi}(t, x, y, z)=\left(\begin{array}{c}
\partial_{t} \operatorname{Re} \psi^{I}(t, x+\mathrm{i} y, \mathrm{i} z) \\
\partial_{t} \operatorname{Im} \psi^{I}(t, x+\mathrm{i} y, \mathrm{i} z) \\
\partial_{t} \mathrm{e}^{\beta_{J}^{\top} t} z
\end{array}\right)
\end{aligned}
$$

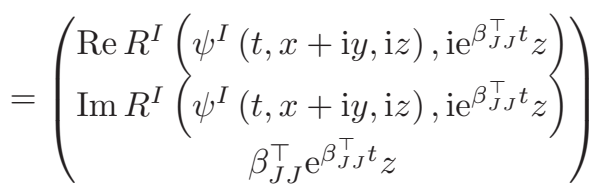

$$
\begin{aligned}
& =\left(\begin{array}{c}
\operatorname{Re} R^{I}\left(\operatorname{Re} \psi^{I}(t, x+\mathrm{i} y, \mathrm{i} z)+\mathrm{i} \operatorname{Im} \psi^{I}(t, x+\mathrm{i} y, \mathrm{i} z), \mathrm{ie}^{\beta_{J J}^{\top} t} z\right) \\
\operatorname{Im} R^{I}\left(\operatorname{Re} \psi^{I}(t, x+\mathrm{i} y, \mathrm{i} z)+\mathrm{i} \operatorname{im} \psi^{I}(t, x+\mathrm{i} y, \mathrm{i} z), \mathrm{ie}^{\beta_{J}^{\top} t} z\right)
\end{array}\right) \\
& =\left(\begin{array}{c}
\operatorname{Re} R^{I}(\vartheta+\mathrm{i} \eta, \mathrm{i} \zeta) \\
\operatorname{Im} R^{I}(\vartheta+\mathrm{i} \eta, \mathrm{i} \zeta) \\
\beta_{J J}^{\top} \zeta
\end{array}\right) \\
& =: \widetilde{R}(\vartheta, \eta, \zeta),
\end{aligned}
$$

where the map $\mathbb{R}_{\leqslant 0}^{m} \times \mathbb{R}^{m} \times \mathbb{R}^{n} \ni(\vartheta, \eta, \zeta) \mapsto \widetilde{R}(\vartheta, \eta, \zeta)$ is $C^{1}$ by [8, Lemma 5.3]. Hence $\widetilde{\psi}(t, x, y, z)$ solves the equation

$$
\partial_{t} \widetilde{\psi}(t, x, y, z)=\widetilde{R}(\widetilde{\psi}(t, x, y, z)), \quad t \geqslant 0, \quad \psi(0, x, y, z)=(x, y, z) .
$$

Similarly to [8, p.1011, (6.7)], we have, for $u=(x+\mathrm{i} y, \mathrm{i} z)$,

$$
\begin{aligned}
\operatorname{Re} R_{i}(x+\mathrm{i} y, \mathrm{i} z)= & \alpha_{i, i i} x_{i}^{2}-\left\langle\alpha_{i} \operatorname{Im} u, \operatorname{Im} u\right\rangle+\sum_{k=1}^{m} \beta_{k i} x_{k} \\
& +\int_{D \backslash\{0\}}\left(\mathrm{e}^{\left\langle\xi_{I}, x\right\rangle} \cos \langle\operatorname{Im} u, \xi\rangle-1-\left\langle\xi_{I}, x\right\rangle\right) \mu_{i}(\mathrm{~d} \xi)
\end{aligned}
$$

and

$\operatorname{Im} R_{i}(x+\mathrm{i} y, \mathrm{i} z)=2 \alpha_{i, i i} x_{i} y_{i}+\left\langle\beta_{I i}, y\right\rangle+\left\langle\beta_{J i}, z\right\rangle$

$$
+\int_{D \backslash\{0\}}\left(\mathrm{e}^{\left\langle\xi_{I}, x\right\rangle} \sin \langle\operatorname{Im} u, \xi\rangle-\langle\operatorname{Im} u, \xi\rangle\right) \mu_{i}(\mathrm{~d} \xi) .
$$

Since $\widetilde{R}: \mathbb{R}_{\leqslant 0}^{m} \times \mathbb{R}^{m+n} \rightarrow \mathbb{R}^{2 m+n}$ is $C^{1}$, so

$$
\left\|\widetilde{R}(\vartheta, \eta, \zeta)-D \widetilde{R}(\mathbf{0})(\vartheta, \eta, \zeta)^{\top}\right\|
$$




$$
\begin{aligned}
& =\left\|\widetilde{R}(\vartheta, \eta, \zeta)-\widetilde{R}(\mathbf{0})-D \widetilde{R}(\mathbf{0})(\vartheta, \eta, \zeta)^{\top}\right\| \\
& =\left\|\int_{0}^{1} D \widetilde{R}(r(\vartheta, \eta, \zeta))(\vartheta, \eta, \zeta)^{\top} \mathrm{d} r-\int_{0}^{1} D \widetilde{R}(\mathbf{0})(\vartheta, \eta, \zeta)^{\top} \mathrm{d} r\right\| \\
& \leqslant \sup _{0 \leqslant r \leqslant 1}\|D \widetilde{R}(r(\vartheta, \eta, \zeta))-D \widetilde{R}(\mathbf{0})\| \cdot\left\|(\vartheta, \eta, \zeta)^{\top}\right\| \\
& =o\left(\left\|(\vartheta, \eta, \zeta)^{\top}\right\|\right)
\end{aligned}
$$

holds. Here, $D \widetilde{R}(\vartheta, \eta, \zeta)$ denotes the Jacobian, i.e., the matrix consisting of all first-order partial derivatives of the vector-valued function $(\vartheta, \eta, \zeta) \mapsto \widetilde{R}(\vartheta, \eta, \zeta)$. According to (3.35) and (3.36), we see that $D \widetilde{R}(\mathbf{0})$ is a matrix taking the form

$$
D \widetilde{R}(\mathbf{0})=\left(\begin{array}{cc:c}
\beta_{I I}^{\top} & 0 & 0 \\
0 & \beta_{I I}^{\top} & * \\
\hdashline 0 & 0 & \beta_{J J}^{\top}
\end{array}\right)
$$

where $*$ is a $(m \times n)$-matrix. By the Riccati equation (3.34) for $\widetilde{\psi}$, we can write

$$
\partial_{t} \widetilde{\psi}(t, x, y, z)=D \widetilde{R}(\mathbf{0}) \widetilde{\psi}(t, x, y, z)+(\widetilde{R}(\widetilde{\psi}(t, x, y, z))-D \widetilde{R}(\mathbf{0}) \widetilde{\psi}(t, x, y, z)) .
$$

From (3.37) it follows that

$$
\lim _{\|(\vartheta, \eta, \zeta)\| \rightarrow 0} \frac{\left\|\widetilde{R}(\vartheta, \eta, \zeta)-D \widetilde{R}(\mathbf{0})(\vartheta, \eta, \zeta)^{\top}\right\|}{\|(\vartheta, \eta, \zeta)\|}=0 .
$$

By assumption, we know that $\beta_{I I} \in \mathbb{M}_{m}^{-}$and $\beta_{J J} \in \mathbb{M}_{n}^{-}$, which ensures $D \widetilde{R}(\mathbf{0}) \in$ $\mathbb{M}_{2 m+n}^{-}$. Now, an application of the linearized stability theorem (see, e.g., 26, VII. Stability Theorem, p.311]) yields that $\widetilde{\psi}$ is asymptotically stable at $\mathbf{0}$. Moreover, as shown in the proof of [26. VII. Stability Theorem, p.311], we can find constants $\delta, c_{1}, c_{2}>0$ such that

$$
\|\widetilde{\psi}(t, x, y, z)\| \leqslant c_{1} \mathrm{e}^{-c_{2} t}, \quad \forall t \geqslant 0,(x, y, z) \in B_{\delta}(0) \cap \mathbb{R}_{\leqslant 0}^{m} \times \mathbb{R}^{m+n},
$$

where $B_{\delta}(0)$ denotes the ball with center 0 and radius $\delta$. By the definition of $\widetilde{\psi}$, the latter inequality implies that (3.33) is true. The lemma is proved.

Next, we extend the estimate in Lemma 3.9 to all $u \in \mathcal{U}$.

Proposition 3.10. Let $X$ be an affine process satisfying (3.1). Suppose that $\beta \in \mathbb{M}_{d}^{-}$. Then for every $u \in \mathcal{U}$, there exist positive constants $c_{1}, c_{2}$, which depend on $u$, such that

$$
\|\psi(t, u)\| \leqslant c_{1} \exp \left\{-c_{2} t\right\}, \quad t \geqslant 0 .
$$

Proof. Our proof is inspired by the proof of [12, Theorem 2.4]. By Proposition 3.8. we have $\sup _{t \in \mathbb{R}_{\geqslant 0}} \mathbb{E}_{x}\left[\left\|X_{t}\right\|\right]<\infty$ for all $x \in D$. Then for $M>0$,

$$
\mathbb{P}_{x}\left(\left\|X_{t}\right\|>M\right) \leqslant \frac{\mathbb{E}_{x}\left[\left\|X_{t}\right\|\right]}{M} \leqslant \frac{\sup _{t \geqslant 0} \mathbb{E}_{x}\left[\left\|X_{t}\right\|\right]}{M},
$$


which implies

$$
\sup _{t \geqslant 0} \mathbb{P}_{x}\left(\left\|X_{t}\right\|>M\right) \rightarrow 0 \quad \text { as } M \rightarrow \infty .
$$

We see that under $\mathbb{P}_{x}$, the sequence $\left\{X_{t}, t \geqslant 0\right\}$ is tight. Consider an arbitrary subsequence $\left\{X_{t^{\prime}}\right\}$. Then it contains a further subsequence $\left\{X_{t^{\prime \prime}}\right\}$ converging in law to some limiting random vector, say $X^{a}$. Since $X_{t^{\prime \prime}}$ converges weakly to $X^{a}$ as $t^{\prime \prime} \rightarrow \infty$, Lévy's continuity theorem implies that the characteristic function of $X_{t^{\prime \prime}}$ converges pointwise to that of $X^{a}$, namely,

$$
\lim _{t^{\prime \prime} \rightarrow \infty} \mathbb{E}_{x}\left[\exp \left\{\left\langle u, X_{t^{\prime \prime}}\right\rangle\right\}\right]=\mathbb{E}\left[\exp \left\{\left\langle u, X^{a}\right\rangle\right\}\right], \quad \text { for all } u \in \mathcal{U} .
$$

We know by Proposition 3.9 that the original sequence $\left\{X_{t}\right\}$ satisfies

$$
\lim _{t \rightarrow \infty} \mathbb{E}_{x}\left[\exp \left\{\left\langle u, X_{t}\right\rangle\right\}\right]=\lim _{t \rightarrow \infty} \exp \{\langle x, \psi(t, u)\rangle\}=1
$$

for all $u \in \mathcal{U}$ with $\|u\|<\delta$. As a consequence, we get

$$
\mathbb{E}\left[\exp \left\{\left\langle u, X^{a}\right\rangle\right\}\right]=1, \quad \text { for all } u \in \mathcal{U} \quad \text { with }\|u\|<\delta .
$$

We claim that $X^{a}=0$ almost surely. To prove this, we consider an arbitrary $z \in \mathbb{R}^{d}$ with $z \neq 0$. Then there exists an $u_{0} \in \mathbb{R}^{d}$ with $\left\|u_{0}\right\|<\delta$ such that $0<\left\langle u_{0}, z\right\rangle<\pi / 6$, and hence $0<\cos \left(\left\langle u_{0}, z\right\rangle\right)<1$. Continuity of cosinus implies that there exists an $\varepsilon>0$ such that $0 \notin B_{\varepsilon}(z):=\left\{y \in \mathbb{R}^{d}:\|y-z\|<\varepsilon\right\}$ and $0<\cos \left(\left\langle u_{0}, y\right\rangle\right)<1$ for all $y \in B_{\varepsilon}(z)$. Suppose that $\mathbb{P}\left(X^{a} \in B_{\varepsilon}(z)\right)>0$. It follows that

$$
\mathbb{E}\left[\cos \left(\left\langle u_{0}, X^{a}\right\rangle\right) \mathbb{1}_{\left\{X^{a} \in B_{\varepsilon}(z)\right\}}\right]<\mathbb{P}\left(X^{a} \in B_{\varepsilon}(z)\right),
$$

which in turn implies

$$
\begin{aligned}
\operatorname{Re} \mathbb{E}\left[\exp \left\{\mathrm{i}\left\langle u_{0}, X^{a}\right\rangle\right\}\right]= & \mathbb{E}\left[\cos \left(\left\langle u_{0}, X^{a}\right\rangle\right)\right] \\
\leqslant & \mathbb{E}\left[\cos \left(\left\langle u_{0}, X^{a}\right\rangle\right) \mathbb{1}_{\left\{X^{a} \in B_{\varepsilon}(z)\right\}}\right] \\
& +\mathbb{E}\left[\cos \left(\left\langle u_{0}, X^{a}\right\rangle\right) \mathbb{1}_{\left\{X^{a} \notin B_{\varepsilon}(z)\right\}}\right] \\
& <\mathbb{P}\left(X^{a} \in B_{\varepsilon}(z)\right)+\mathbb{P}\left(X^{a} \notin B_{\varepsilon}(z)\right) \\
= & 1,
\end{aligned}
$$

a contradiction to (3.38) . We conclude that $\mathbb{P}\left(X^{a} \in B_{\varepsilon}(z)\right)=0$. Since $z \neq 0$ is arbitrary, $X^{a}$ must be 0 almost surely. Now we have shown that every subsequence of $\left\{X_{t}\right\}$ contains a further subsequence converging weakly to $\delta_{0}$, so the original sequence $\left\{X_{t}\right\}$ must converge to $\delta_{0}$ weakly. In view of this, we now denote $X^{a}$ by $X_{\infty}$ which is 0 almost surely. We have thus shown that for all $x \in D$ and $u \in \mathcal{U}$,

$$
\exp \{\langle x, \psi(t, u)\rangle\}=\mathbb{E}_{x}\left[\exp \left\{\left\langle u, X_{t}\right\rangle\right\}\right] \rightarrow 1 \text { as } t \rightarrow \infty .
$$

From the above convergence of $\exp \{\langle x, \psi(t, u)\rangle\}$ to 1 , we infer that for each $i=1, \ldots, d$,

$$
\operatorname{Re} \psi_{i}(t, u) \rightarrow 0 \quad \text { as } t \rightarrow \infty .
$$

Moreover, we must have $\sup _{t \in[0, \infty)}\left|\psi_{i}(t, u)\right| \leqslant C$ for some constant $C=C(u)<$ $\infty$, otherwise, by continuity, $\operatorname{Im} \psi_{i}(t, u)$ hits the set $\{2 k \pi+\pi / 2: k \in \mathbb{Z}\}$ infinitely many times as $t \rightarrow \infty$, so $\sin \left(\operatorname{Im} \psi_{i}(t, u)\right)=1$ infinitely often, contradicting the fact that $\exp \{\langle x, \psi(t, u)\rangle\} \rightarrow 1$ for all $x \in D$. 
Let $z, z^{\prime} \in \mathbb{C}$ be two different accumulation points of $\left\{\psi_{1}(t, u), t \geqslant 0\right\}$ as $t \rightarrow \infty$, that is, we can find sequences $t_{n}, t_{n}^{\prime} \rightarrow \infty$ such that $\psi_{1}\left(t_{n}, u\right) \rightarrow z$ and $\psi_{1}\left(t_{n}^{\prime}, u\right) \rightarrow z^{\prime}$. Using once again the convergence in (3.39), we obtain that $z=\mathrm{i} 2 \pi k_{1}$ and $z^{\prime}=\mathrm{i} 2 \pi k_{2}$ for some $k_{1}, k_{2} \in \mathbb{Z}$. By (3.40) and a similar argument as in the last paragraph, $\psi_{1}(t, u)$ is not allowed to fluctuate between $z$ and $z^{\prime}$, showing that $z=z^{\prime}$. So $z=\mathrm{i} 2 \pi k_{1}$ is the only accumulation point of $\left\{\psi_{1}(t, u), t \geqslant 0\right\}$, and $\psi_{1}(t, u) \rightarrow z=\mathrm{i} 2 \pi k_{1}$ as $t \rightarrow \infty$. Moreover, we must have $k_{1}=0$, otherwise for some $x \in D$ we get $\exp \left\{x_{1} 2 \pi \mathrm{i} k_{1}\right\} \neq 1$, which is impossible due to (3.39). We conclude that

$$
\psi_{1}(t, u) \rightarrow 0 \quad \text { as } t \rightarrow \infty \text { for all } u \in \mathcal{U} \text {. }
$$

In the same way it follows that $\psi_{i}(t, u) \rightarrow 0$ as $t \rightarrow 0$ for all $i=2, \ldots, d$ and $u \in \mathcal{U}$.

Finally, we prove that the convergence of $\psi(t, u)$ to zero as $t \rightarrow \infty$ is exponentially fast. Since $\psi(t, u)$ converges to 0 as $t \rightarrow \infty$, there exists a $t_{0}>0$ such that $\left\|\psi\left(t_{0}, u\right)\right\|<\delta$. Combining Lemma 3.9 with the semi-flow property of $\psi$, we conclude that

$$
\left\|\psi\left(t+t_{0}, u\right)\right\|=\left\|\psi\left(t, \psi\left(t_{0}, u\right)\right)\right\| \leqslant c_{1} \mathrm{e}^{-c_{2} t}, \quad t \geqslant 0
$$

for some positive constants $c_{1}$ and $c_{2}$. Hence,

$$
\|\psi(t, u)\| \leqslant c_{3} \mathrm{e}^{-c_{2} t}, \quad t \geqslant t_{0} .
$$

Since $\sup _{t \in\left[0, t_{0}\right]}\|\psi(t, u)\|<c_{4}$, where $c_{4}>0$ is a constant, it follows that

$$
\|\psi(t, u)\| \leqslant c_{5} \mathrm{e}^{-c_{2} t}, \quad t \geqslant 0
$$

with another constant $c_{5}>0$. This completes our proof.

\section{Proof of the main result}

In this section we will prove Theorem 2.6

Let $X$ be an affine process with state space $D$ and admissible parameters $(a, \alpha, b, \beta, m, \mu)$. Recall that $F(u)$ is given by (2.7). We start with the following lemma.

Lemma 4.1. Suppose $\beta \in \mathbb{M}_{d}^{-}$and $\int_{\{\|\xi\|>1\}} \log \|\xi\| m(\mathrm{~d} \xi)<\infty$. Then

$$
\int_{0}^{\infty}|F(\psi(s, u))| \mathrm{d} s<\infty \quad \text { for all } u \in \mathcal{U} .
$$

Proof. Let $u \in \mathcal{U}$ be fixed. By Remark 3.1 and Proposition 3.10, we can find constants $c_{1}, c_{2}>0$ depending on $u$ such that

$$
\|\psi(s, u)\| \leqslant c_{1} \mathrm{e}^{-c_{2} s}, \quad s \geqslant 0 .
$$

It is clear that finiteness of $\int_{0}^{\infty}|F(\psi(s, u))| \mathrm{d} s$ depends only on the jump part of $F$. We define

$$
\begin{aligned}
\mathcal{I}(u)= & \int_{0}^{\infty} \int_{\{0<\|\xi\| \leqslant 1\}}\left|\mathrm{e}^{\langle\xi, \psi(s, u)\rangle}-1-\left\langle\psi^{J}(s, u), \xi_{J}\right\rangle\right| m(\mathrm{~d} \xi) \mathrm{d} s \\
& +\int_{0}^{\infty} \int_{\{\|\xi\|>1\}}\left|\mathrm{e}^{\langle\xi, \psi(s, u)\rangle}-1\right| m(\mathrm{~d} \xi) \mathrm{d} s
\end{aligned}
$$




$$
=: \mathcal{I}_{*}(u)+\mathcal{I}^{*}(u) .
$$

With the latter fact in mind, we start with the big jumps. We can apply Fubini's theorem to get

$$
\mathcal{I}^{*}(u)=\int_{\{\|\xi\|>1\}} \int_{0}^{\infty}\left|\mathrm{e}^{\langle\xi, \psi(s, u)\rangle}-1\right| \mathrm{d} s m(\mathrm{~d} \xi) .
$$

Let us define $I_{1}(\xi):=\int_{0}^{\infty}|\exp \{\langle\psi(s, u), \xi\rangle\}-1| \mathrm{d} s$. For $\|\xi\|>1$, by a change of variables $t:=\exp \left\{-c_{2} s\right\}\|\xi\|$, we get $\mathrm{d} s=-c_{2}^{-1} t^{-1} \mathrm{~d} t$, and hence

$$
\begin{aligned}
I_{1}(\xi) & =-\frac{1}{c_{2}} \int_{\|\xi\|}^{0} \frac{1}{t}\left|\mathrm{e}^{\left\langle\xi, \psi\left(s^{-1}(t), u\right)\right\rangle}-1\right| \mathrm{d} t \\
& =\frac{1}{c_{2}} \int_{0}^{\|\xi\|} \frac{1}{t}\left|\mathrm{e}^{\left\langle\xi, \psi\left(s^{-1}(t), u\right)\right\rangle}-1\right| \mathrm{d} t \\
& \leqslant \frac{1}{c_{2}} \int_{0}^{1} \frac{1}{t}\left|\mathrm{e}^{\left\langle\xi, \psi\left(s^{-1}(t), u\right)\right\rangle}-1\right| \mathrm{d} t+\frac{1}{c_{2}} \int_{1}^{\|\xi\|} \frac{2}{t} \mathrm{~d} t \\
& =: I_{2}(\xi)+I_{3}(\xi) .
\end{aligned}
$$

Note that

$$
\begin{aligned}
\left|\mathrm{e}^{\left\langle\xi, \psi\left(s^{-1}(t), u\right)\right\rangle}-1\right| & =\left|\int_{0}^{1} \mathrm{e}^{r\left\langle\xi, \psi\left(s^{-1}(t), u\right)\right\rangle}\left\langle\xi, \psi\left(s^{-1}(t), u\right)\right\rangle \mathrm{d} r\right| \\
& \leqslant\left|\left\langle\xi, \psi\left(s^{-1}(t), u\right)\right\rangle\right| .
\end{aligned}
$$

Using (4.1), we obtain

$$
\begin{aligned}
I_{2}(\xi) & \leqslant \frac{1}{c_{2}} \int_{0}^{1} \frac{1}{t}\left|\left\langle\psi\left(s^{-1}(t), u\right), \xi\right\rangle\right| \mathrm{d} t \\
& \leqslant \frac{1}{c_{2}} \int_{0}^{1} \frac{1}{t}\left\|\psi\left(s^{-1}(t), u\right)\right\|\|\xi\| \mathrm{d} t \\
& \leqslant \frac{1}{c_{2}} \int_{0}^{1} \frac{c_{1}}{t} \mathrm{e}^{-c_{2} s^{-1}(t)}\|\xi\| \mathrm{d} t .
\end{aligned}
$$

Since $s^{-1}(t)=\log \left(t\|\xi\|^{-1}\right)\left(-c_{2}\right)^{-1}$, it follows that

$$
I_{2}(\xi) \leqslant \frac{1}{c_{2}} \int_{0}^{1} c_{1} \mathrm{~d} t=\frac{c_{1}}{c_{2}}
$$

On the other hand, it is easy to see that

$$
I_{3}(\xi) \leqslant \frac{2}{c_{2}} \log \|\xi\|,
$$

Having established the latter inequalities, we conclude that

$$
\begin{aligned}
\left|\mathcal{I}^{*}(u)\right| & \leqslant \int_{\{\|\xi\|>1\}}\left(I_{2}(\xi)+I_{3}(\xi)\right) m(\mathrm{~d} \xi) \\
& \leqslant \int_{\{\|\xi\|>1\}}\left(\frac{c_{1}}{c_{2}}+\frac{2}{c_{2}} \log \|\xi\|\right) m(\mathrm{~d} \xi) \\
& =\frac{c_{1}}{c_{2}} m(\{\|\xi\|>1\})+\frac{2}{c_{2}} \int_{\{\|\xi\|>1\}} \log \|\xi\| m(\mathrm{~d} \xi) .
\end{aligned}
$$


Because the Lévy measure $m(\mathrm{~d} \xi)$ integrates $\mathbb{1}_{\{\|\xi\|>1\}} \log \|\xi\|$ by assumption, we see that

$$
\mathcal{I}^{*}(u)<\infty \text {. }
$$

We now turn to $\mathcal{I}_{*}(\xi)$. We can write

$$
\begin{aligned}
\mathrm{e}^{\langle\xi, \psi(s, u)\rangle}- & 1-\left\langle\psi^{J}(s, u), \xi_{J}\right\rangle \\
= & \int_{0}^{1} \mathrm{e}^{r\langle\xi, \psi(s, u)\rangle}\langle\psi(s, u), \xi\rangle \mathrm{d} r-\left\langle\psi^{J}(s, u), \xi_{J}\right\rangle \\
= & \int_{0}^{1} \mathrm{e}^{r\langle\xi, \psi(s, u)\rangle}\left\langle\psi^{I}(s, u), \xi_{I}\right\rangle \mathrm{d} r+\int_{0}^{1}\left(\mathrm{e}^{r\langle\xi, \psi(s, u)\rangle}-1\right)\left\langle\psi^{J}(s, u), \xi_{J}\right\rangle \mathrm{d} r \\
= & \int_{0}^{1} \mathrm{e}^{r\langle\xi, \psi(s, u)\rangle}\left\langle\psi^{I}(s, u), \xi_{I}\right\rangle \mathrm{d} r \\
& \quad+\int_{0}^{1} \int_{0}^{1} \mathrm{e}^{r r^{\prime}\langle\xi, \psi(s, u)\rangle} r\langle\xi, \psi(s, u)\rangle\left\langle\psi^{J}(s, u), \xi_{J}\right\rangle \mathrm{d} r \mathrm{~d} r^{\prime} .
\end{aligned}
$$

Noting (4.1) and $\operatorname{Re}(\langle\xi, \psi(s, u)\rangle) \leqslant 0$, we deduce that for $\|\xi\| \leqslant 1$ and $s \geqslant 0$,

$$
\begin{aligned}
\left|\mathrm{e}^{\langle\xi, \psi(s, u)\rangle}-1-\left\langle\psi^{J}(s, u), \xi_{J}\right\rangle\right| & \leqslant\left\|\psi^{I}(s, u)\right\|\left\|\xi_{I}\right\|+\|\psi(s, u)\|\|\xi\|\left\|\psi^{J}(s, u)\right\|\left\|\xi_{J}\right\| \\
& \leqslant\left(c_{1}+c_{1}^{2}\right) \mathrm{e}^{-c_{2} s}\left(\left\|\xi_{I}\right\|+\left(\left\|\xi_{I}\right\|+\left\|\xi_{J}\right\|\right)\left\|\xi_{J}\right\|\right) \\
& \leqslant\left(c_{1}+c_{1}^{2}\right) \mathrm{e}^{-c_{2} s}\left(2\left\|\xi_{I}\right\|+\left\|\xi_{J}\right\|^{2}\right) .
\end{aligned}
$$

So

$$
\mathcal{I}_{*}(u) \leqslant\left(c_{1}+c_{1}^{2}\right) \int_{0}^{\infty} \mathrm{e}^{-c_{2} s} \mathrm{~d} s \int_{\{0<\|\xi\| \leqslant 1\}}\left(2\left\|\xi_{I}\right\|+\left\|\xi_{J}\right\|^{2}\right) m(\mathrm{~d} \xi)<\infty,
$$

where the finiteness of the integral on the right-hand side follows by Definition 2.2 (iii). Since (4.2) holds, it follows that

$$
\int_{0}^{\infty}|F(\psi(s, u))| \mathrm{d} s \leqslant \mathcal{I}(u)=\mathcal{I}_{*}(u)+\mathcal{I}^{*}(u)<\infty .
$$

The lemma is proved.

We are now ready to prove our main result.

Proof of Theorem [2.6. Recall that the characteristic function of $X_{t}$ is given by

$$
\mathbb{E}_{x}\left[\mathrm{e}^{\left\langle u, X_{t}\right\rangle}\right]=\exp \{\phi(t, u)+\langle x, \psi(t, u)\rangle\}, \quad(t, u) \in \mathbb{R}_{\geqslant 0} \times \mathcal{U} .
$$

Using Remark 3.1. Theorem 3.10 and Lemma 4.1, we have that $\psi(t, u) \rightarrow 0$ and

$$
\phi(t, u)=\int_{0}^{t} F(\psi(s, u)) \mathrm{d} s \rightarrow \int_{0}^{\infty} F(\psi(s, u)) \mathrm{d} s, \quad \text { as } t \rightarrow \infty .
$$

We now verify that $\int_{0}^{\infty} F(\psi(s, u)) \mathrm{d} s$ is continuous at $u=0$. It is easy to see that that $\int_{0}^{T} F(\psi(s, u)) \mathrm{d} s$ is continuous at $u=0$. It suffices to show that the convergence $\lim _{T \rightarrow \infty} \int_{0}^{T} F(\psi(s, u)) \mathrm{d} s=\int_{0}^{\infty} F(\psi(s, u)) \mathrm{d} s$ is uniform for $u$ in a 
small neighborhood of 0 . By (3.33), there exist $\delta>0$ and constants $c_{1}, c_{2}>0$ such that for all $B_{\delta}(0) \cap \mathcal{U}$,

$$
\|\psi(t, u)\| \leqslant c_{1} \exp \left\{-c_{2} t\right\}, \quad t \geqslant 0 .
$$

Define

$$
\begin{aligned}
\mathcal{I}_{T}(u)= & \int_{T}^{\infty} \int_{\{0<\|\xi\| \leqslant 1\}}\left|\mathrm{e}^{\langle\xi, \psi(s, u)\rangle}-1-\left\langle\psi^{J}(s, u), \xi_{J}\right\rangle\right| m(\mathrm{~d} \xi) \mathrm{d} s \\
& +\int_{T}^{\infty} \int_{\{1<\|\xi\| \leqslant K\}}\left|\mathrm{e}^{\langle\xi, \psi(s, u)\rangle}-1\right| m(\mathrm{~d} \xi) \mathrm{d} s \\
& +\int_{T}^{\infty} \int_{\{\|\xi\|>K\}}\left|\mathrm{e}^{\langle\xi, \psi(s, u)\rangle}-1\right| m(\mathrm{~d} \xi) \mathrm{d} s \\
= & : \mathcal{I}_{*, T}(u)+\mathcal{I}_{T}^{*}(u)+\mathcal{I}_{T}^{* *}(u),
\end{aligned}
$$

where $K>0$. Let $\varepsilon>0$ be arbitrary. By Fubini's theorem,

$$
\mathcal{I}_{T}^{* *}(u)=\int_{\{\|\xi\|>K\}} \int_{T}^{\infty}\left|\mathrm{e}^{\langle\xi, \psi(s, u)\rangle}-1\right| \mathrm{d} s m(\mathrm{~d} \xi) .
$$

Set $I_{1}(\xi):=\int_{T}^{\infty}|\exp \{\langle\psi(s, u), \xi\rangle\}-1| \mathrm{d} s$. As in the proof of Lemma 4.1 we introduce a change of variables $t:=\exp \left\{-c_{2}(s-T)\right\}\|\xi\|$ and obtain for $\|\xi\|>1$,

$$
\begin{aligned}
I_{1}(\xi) & =\frac{1}{c_{2}} \int_{0}^{\|\xi\|} \frac{1}{t}\left|\mathrm{e}^{\left\langle\xi, \psi\left(s^{-1}(t), u\right)\right\rangle}-1\right| \mathrm{d} t \\
& \leqslant \frac{1}{c_{2}} \int_{0}^{1} \frac{1}{t}\left|\mathrm{e}^{\left\langle\xi, \psi\left(s^{-1}(t), u\right)\right\rangle}-1\right| \mathrm{d} t+\frac{1}{c_{2}} \int_{1}^{\|\xi\|} \frac{2}{t} \mathrm{~d} t \\
& \leqslant \frac{1}{c_{2}} \int_{0}^{1} \frac{c_{1}}{t} \mathrm{e}^{-c_{2} s^{-1}(t)}\|\xi\| \mathrm{d} t+\frac{2}{c_{2}} \log \|\xi\| \\
& \leqslant \frac{1}{c_{2}} \int_{0}^{1} c_{1} e^{-c_{2} T} \mathrm{~d} t+\frac{2}{c_{2}} \log \|\xi\| .
\end{aligned}
$$

So

$$
\begin{aligned}
\mathcal{I}_{T}^{* *}(u) & \leqslant \int_{\{\|\xi\|>K\}}\left(\frac{c_{1}}{c_{2}} e^{-c_{2} T}+\frac{2}{c_{2}} \log \|\xi\|\right) m(\mathrm{~d} \xi) \\
& \leqslant \frac{c_{1}}{c_{2}} m(\{\|\xi\|>K\})+\frac{2}{c_{2}} \int_{\{\|\xi\|>K\}} \log \|\xi\| m(\mathrm{~d} \xi) .
\end{aligned}
$$

We now choose $K>0$ large enough such that $\mathcal{I}_{T}^{* *}(u)<\varepsilon / 3$.

For $\mathcal{I}_{T}^{*}(u)$, by (4.4), we have

$$
\begin{aligned}
I_{1}(\xi) & =\frac{1}{c_{2}} \int_{0}^{\|\xi\|} \frac{1}{t}\left|\mathrm{e}^{\left\langle\xi, \psi\left(s^{-1}(t), u\right)\right\rangle}-1\right| \mathrm{d} t \\
& \leqslant \frac{1}{c_{2}} \int_{0}^{\|\xi\|} \frac{c_{1}}{t} \mathrm{e}^{-c_{2} s^{-1}(t)}\|\xi\| \mathrm{d} t \\
& \leqslant \frac{1}{c_{2}} \int_{0}^{\|\xi\|} c_{1} e^{-c_{2} T} \mathrm{~d} t
\end{aligned}
$$




$$
\leqslant \frac{c_{1}}{c_{2}} e^{-c_{2} T}\|\xi\|
$$

which imples

$$
\begin{aligned}
\mathcal{I}_{T}^{*}(u) & \leqslant \int_{\{1<\|\xi\| \leq K\}}\left(\frac{c_{1}}{c_{2}} e^{-c_{2} T}\|\xi\|\right) m(\mathrm{~d} \xi) \\
& \leqslant \frac{c_{1}}{c_{2}} e^{-c_{2} T} \int_{\{1<\|\xi\| \leq K\}}\|\xi\| m(\mathrm{~d} \xi) \rightarrow 0, \quad \text { as } T \rightarrow \infty .
\end{aligned}
$$

So we find $T_{1}>0$ such that for $T>T_{1}, \mathcal{I}_{T}^{*}(u)<\varepsilon / 3$. It follows from (4.3) that $\mathcal{I}_{*, T}(u) \leqslant\left(c_{1}+c_{1}^{2}\right) \int_{T}^{\infty} \mathrm{e}^{-c_{2} s} \mathrm{~d} s \int_{\{0<\|\xi\| \leqslant 1\}}\left(2\left\|\xi_{I}\right\|+\left\|\xi_{J}\right\|^{2}\right) m(\mathrm{~d} \xi) \rightarrow 0, \quad$ as $T \rightarrow \infty$.

Hence there exists $T_{2}>T_{1}$ such that for $T>T_{2}, \mathcal{I}_{*, T}(u)<\varepsilon / 3$. Finally, we get for $T>T_{2}$,

$$
\int_{T}^{\infty}|F(\psi(s, u))| \mathrm{d} s \leqslant \mathcal{I}_{*, T}(u)+\mathcal{I}_{T}^{*}(u)+\mathcal{I}_{T}^{* *}(u)<\varepsilon
$$

Moreover, the particular choice of above $K, T_{1}, T_{2}$ do not depend on $u \in B_{\delta}(0) \cap \mathcal{U}$. We thus obtain the desired uniform convergence and further the continuity of $\int_{0}^{\infty} F(\psi(s, u)) \mathrm{d} s$ at $u=0$.

By Lévy's continuity theorem, the limiting distribution of $X_{t}$ exists and we denote it by $\pi$. The limiting distribution $\pi$ has characteristic function

$$
\int_{D} \mathrm{e}^{\langle u, x\rangle} \pi(\mathrm{d} x)=\exp \left\{\int_{0}^{\infty} F(\psi(s, u)) \mathrm{d} s\right\} .
$$

We now verify that $\pi$ is the unique stationary distribution. We start with the stationarity. Suppose that $X_{0}$ is distributed according to $\pi$. Then, for any $u \in \mathcal{U}$,

$$
\begin{aligned}
\mathbb{E}_{\pi}\left[\exp \left\{\left\langle u, X_{t}\right\rangle\right\}\right] & =\int_{D} \exp \{\phi(t, u)+\langle x, \psi(t, u)\rangle\} \pi(\mathrm{d} x) \\
& =\mathrm{e}^{\phi(t, u)} \int_{D} \exp \{\langle x, \psi(t, u)\rangle\} \pi(\mathrm{d} x) \\
& =\mathrm{e}^{\phi(t, u)} \int_{D} \mathrm{e}^{\langle x, \eta\rangle} \pi(\mathrm{d} x),
\end{aligned}
$$

where we substituted $\eta:=\psi(t, u)$ in the last equality. Note that the integral on the right-hand side of the last equality is the characteristic function of the limit distribution $\pi$. Therefore, using the semi-flow property of $\psi$ in (2.3), we have

$$
\begin{aligned}
\mathbb{E}_{\pi}\left[\exp \left\{\left\langle u, X_{t}\right\rangle\right\}\right] & =\mathrm{e}^{\phi(t, u)} \exp \left\{\int_{0}^{\infty} F(\psi(s, \eta)) \mathrm{d} s\right\} \\
& =\mathrm{e}^{\phi(t, u)} \exp \left\{\int_{0}^{\infty} F(\psi(s, \psi(t, u))) \mathrm{d} s\right\} \\
& =\mathrm{e}^{\phi(t, u)} \exp \left\{\int_{0}^{\infty} F(\psi(t+s, u)) \mathrm{d} s\right\} \\
& =\mathrm{e}^{\phi(t, u)} \exp \left\{\int_{t}^{\infty} F(\psi(s, u)) \mathrm{d} s\right\} .
\end{aligned}
$$


So, by the generalized Riccati equation (2.5) for $\phi$,

$$
\mathbb{E}_{\pi}\left[\exp \left\{\left\langle u, X_{t}\right\rangle\right\}\right]=\exp \left\{\int_{0}^{\infty} F(\psi(s, u)) \mathrm{d} s\right\}=\int_{D} \mathrm{e}^{\langle x, u\rangle} \pi(\mathrm{d} x) .
$$

Hence $\pi$ is a stationary distribution for $X$.

Finally, we prove the uniqueness of stationary distributions for $X$. We proceed as in [15, p.80]. Suppose that there exists another stationary distribution $\pi^{\prime}$. Let $X_{0}$ be distributed according to $\pi^{\prime}$. Recall that for all $u \in \mathcal{U}, \psi(t, u) \rightarrow 0$ as $t \rightarrow \infty$ in virtue of Theorem 3.10 and, by Lemma 4.1] $\phi(t, u) \rightarrow \int_{0}^{\infty} F(\psi(t, u)) \mathrm{d} s$ as $t \rightarrow \infty$. Hence, by dominated convergence,

$$
\begin{aligned}
\int_{D} \mathrm{e}^{\langle x, u\rangle} \pi^{\prime}(\mathrm{d} x) & =\lim _{t \rightarrow \infty} \mathbb{E}_{\pi^{\prime}}\left[\exp \left\{\left\langle u, X_{t}\right\rangle\right\}\right] \\
& =\lim _{t \rightarrow \infty} \int_{D} \exp \{\phi(t, u)+\langle x, \psi(t, u)\rangle\} \pi^{\prime}(\mathrm{d} x) \\
& =\int_{D} \exp \left\{\int_{0}^{\infty} F(\psi(s, u)) \mathrm{d} s\right\} \pi^{\prime}(\mathrm{d} x) \\
& =\exp \left\{\int_{0}^{\infty} F(\psi(s, u)) \mathrm{d} s\right\}=\int_{D} \mathrm{e}^{\langle x, u\rangle} \pi(\mathrm{d} x) .
\end{aligned}
$$

So $\pi=\pi^{\prime}$.

Acknowledgement. We would like to thank Martin Friesen for several helpful discussions.

\section{References}

1. Leif B. G. Andersen and Vladimir V. Piterbarg, Moment explosions in stochastic volatility models, Finance Stoch. 11 (2007), no. 1, 29-50. MR 2284011

2. Mátyás Barczy, Leif Döring, Zenghu Li, and Gyula Pap, Stationarity and ergodicity for an affine two-factor model, Adv. in Appl. Probab. 46 (2014), no. 3, 878-898. MR 3254346

3. Mátyás Barczy, Zenghu Li, and Gyula Pap, Stochastic differential equation with jumps for multi-type continuous state and continuous time branching processes with immigration, ALEA Lat. Am. J. Probab. Math. Stat. 12 (2015), no. 1, 129-169. MR 3340375

4. Moment formulas for multitype continuous state and continuous time branching process with immigration, J. Theoret. Probab. 29 (2016), no. 3, 958-995. MR 3540486

5. John C. Cox, Jonathan E. Ingersoll, Jr., and Stephen A. Ross, A theory of the term structure of interest rates, Econometrica 53 (1985), no. 2, 385-407.

6. Qiang Dai and Kenneth J. Singleton, Specification analysis of affine term structure models, The Journal of Finance 55 (2000), no. 5, 1943-1978.

7. D. A. Dawson and Zenghu Li, Skew convolution semigroups and affine Markov processes, Ann. Probab. 34 (2006), no. 3, 1103-1142. MR 2243880

8. D. Duffie, D. Filipović, and W. Schachermayer, Affine processes and applications in finance, Ann. Appl. Probab. 13 (2003), no. 3, 984-1053.

9. Darrell Duffie, Jun Pan, and Kenneth Singleton, Transform analysis and asset pricing for affine jump-diffusions, Econometrica 68 (2000), no. 6, 1343-1376. MR 1793362

10. Stewart N. Ethier and Thomas G. Kurtz, Markov processes: Characterization and convergence, Wiley Series in Probability and Mathematical Statistics: Probability and Mathematical Statistics, John Wiley \& Sons, Inc., New York, 1986. MR 838085 (88a:60130)

11. Damir Filipović and Eberhard Mayerhofer, Affine diffusion processes: theory and applications, Advanced financial modelling, Radon Ser. Comput. Appl. Math., vol. 8, Walter de Gruyter, Berlin, 2009, pp. 125-164. MR 2648460 
12. Paul Glasserman and Kyoung-Kuk Kim, Moment explosions and stationary distributions in affine diffusion models, Math. Finance 20 (2010), no. 1, 1-33. MR 2599675

13. Steven L. Heston, A closed-form solution for options with stochastic volatility with applications to bond and currency options, Review of Financial Studies (1993), 6:327?343.

14. Rudra P. Jena, Kyoung-Kuk Kim, and Hao Xing, Long-term and blow-up behaviors of exponential moments in multi-dimensional affine diffusions, Stochastic Process. Appl. 122 (2012), no. 8, 2961-2993. MR 2931348

15. Martin Keller-Ressel, Moment explosions and long-term behavior of affine stochastic volatility models, Math. Finance 21 (2011), no. 1, 73-98. MR 2779872 (2012e:91126)

16. Martin Keller-Ressel and Eberhard Mayerhofer, Exponential moments of affine processes, Ann. Appl. Probab. 25 (2015), no. 2, 714-752. MR 3313754

17. Martin Keller-Ressel and Aleksandar Mijatović, On the limit distributions of continuousstate branching processes with immigration, Stochastic Process. Appl. 122 (2012), no. 6, 2329-2345. MR 2922631

18. Martin Keller-Ressel, Walter Schachermayer, and Josef Teichmann, Affine processes are regular, Probab. Theory Related Fields 151 (2011), no. 3-4, 591-611. MR 2851694 (2012k:60219)

19. John M. Lee, Introduction to smooth manifolds, second ed., Graduate Texts in Mathematics, vol. 218, Springer, New York, 2013. MR 2954043

20. Zenghu Li, Measure-valued branching Markov processes, Probability and its Applications (New York), Springer, Heidelberg, 2011. MR 2760602

21. Eberhard Mayerhofer, Johannes Muhle-Karbe, and Alexander G. Smirnov, A characterization of the martingale property of exponentially affine processes, Stochastic Process. Appl. 121 (2011), no. 3, 568-582. MR 2763096

22. Mark A. Pinsky, Limit theorems for continuous state branching processes with immigration, Bull. Amer. Math. Soc. 78 (1972), 242-244. MR 0295450

23. Ken-iti Sato and Makoto Yamazato, Operator-self-decomposable distributions as limit distributions of processes of Ornstein-Uhlenbeck type, Stochastic Process. Appl. 17 (1984), no. 1, 73-100. MR 738769

24. K. Urbanik, Self-decomposable probability distributions on $R^{m}$, Zastos. Mat. 10 (1969), 9197. MR 0245068

25. Oldrich Vasicek, An equilibrium characterization of the term structure [reprint of J. Financ. Econ. 5 (1977), no. 2, 177-188], Financial risk measurement and management, Internat. Lib. Crit. Writ. Econ., vol. 267, Edward Elgar, Cheltenham, 2012, pp. 724-735. MR 3235239

26. Wolfgang Walter, Ordinary differential equations, Graduate Texts in Mathematics, vol. 182, Springer-Verlag, New York, 1998, Translated from the sixth German (1996) edition by Russell Thompson, Readings in Mathematics. MR 1629775

(Peng Jin) Department of Mathematics, Shantou University, Shantou, Guangdong 515063, CHINA

E-mail address: pjin@stu.edu.cn

(Jonas Kremer) Fakultät für Mathematik und Naturwissenschaften, Bergische Universität Wuppertal, 42119 Wuppertal, Germany

E-mail address: jkremer@uni-wuppertal.de

(Barbara Rüdiger) Fakultät für Mathematik und Naturwissenschaften, Bergische Universität Wuppertal, 42119 Wuppertal, Germany

E-mail address: ruediger@uni-wuppertal.de 